\title{
Some Rational Coupled Fuzzy Cone Contraction Theorems in Fuzzy Cone Metric Spaces with an Application
}

\author{
Saif Ur Rehman $\mathbb{D}^{1},{ }^{1}$ Muhammad Talha Waheed, ${ }^{1}$ Naeem Jan ${ }^{D},{ }^{1}$ Abdu Gumaei ${ }^{D},{ }^{2}$ \\ and Mabrook Al-Rakhami iD ${ }^{3}$ \\ ${ }^{1}$ Department of Mathematics, Gomal University, Dera Ismail Khan 29050, Pakistan \\ ${ }^{2}$ Computer Science Department, Faculty of Applied Sciences, Taiz University, Taiz 6803, Yemen \\ ${ }^{3}$ STC's Artificial Intelligence Chair, Department of Information Systems, College of Computer and Information Sciences, \\ King Saud University, Riyadh 11543, Saudi Arabia
}

Correspondence should be addressed to Abdu Gumaei; abdugumaei@taiz.edu.ye and Mabrook Al-Rakhami; malrakhami@ksu.edu.sa

Received 13 June 2021; Accepted 3 August 2021; Published 18 August 2021

Academic Editor: Huseyin Isik

Copyright (c) 2021 Saif Ur Rehman et al. This is an open access article distributed under the Creative Commons Attribution License, which permits unrestricted use, distribution, and reproduction in any medium, provided the original work is properly cited.

In this paper, we establish the new concept of rational coupled fuzzy cone contraction mapping in fuzzy cone metric spaces and prove some unique rational-type coupled fixed-point theorems in the framework of fuzzy cone metric spaces by using "the triangular property of fuzzy cone metric." To ensure the existence of our results, we present some illustrative unique coupled fixedpoint examples. Furthermore, we present an application of a Lebesgue integral-type contraction mapping in fuzzy cone metric spaces and to prove a unique coupled fixed-point theorem.

\section{Introduction}

In 1965, the theory of fuzzy sets was introduced by Zadeh [1]. Kramosil and Michalek [2] introduced the notion of FMS by using continuous $t$-norm with fuzzy sets. Afterward, Grabiec [3] established the completeness property of the FMS and proved a "Fuzzy Banach Contraction Principle for a unique fixed point (FP) in complete FMS." Since then, many contributed to this theory concerning FP results (e.g., see [4-6]). Later on, in 1994, George and Veeramani [7] modified the concept of FMS introduced by Kramosil and Michalek [2], and they presented the topological properties and proved Baire's theorem on complete FMS. In 2002, some contractive-type FP theorems were proved by Gregory and Sapena [8] on complete FMS by using the concept of [2, 7]. Some related FP concepts in FMSs can be found in [9-12]. Recently, the rational-type fuzzy contraction concept in FMS is given by Rehman et al. [13], and they proved some FP results with an application.
Jaggi [14] proved the rational-type FP result for a contractive condition. However, Harjani et al. [15] modified the concept of Jaggi [14] and proved a generalized result in "partially ordered metric space." In 2011, Luong and Thuan [16] proved generalized rational weak contraction results in "partially ordered metric space," which is a generalization of the result of [14]. In [17], Guo and Lakshmikantham presented the concept of coupled FP with applications by using the nonlinear operator. Later on, Bhaskar [18] and Lakshmikantham [19] proved coupled FP results in "partially ordered metric space.” In [20], Sedghi et al. used commuting mappings and established some common coupled FP theorems in FMSs.

In 2007, the notion of cone metric space (CMS) was introduced by Huang and Zhang [21]. They proved some basic convergence properties and FP theorems on CMS. In 2008, Abbas et al. [22] proved some common FP theorems without continuity for noncommuting mappings on CMS. After that, many others contributed their ideas to the 
problem of FP results in CMS. Some of their FP contributions can be found in [23-25].

Oner et al. [26] introduced the concept of fuzzy cone metric space (FCMS) and proved a "fuzzy cone Banach contraction theorem" for FP in complete FCMSs in which they assumed that the "fuzzy cone contractive ( $f c$ - contractive) sequences are Cauchy." In [27], Rehman and Li proved some FP theorems in FCMSs without the assumption of " $f c$ - contractive sequences are Cauchy" by using the "triangular property of FCM." Some more FP findings in the said space can be found in [28-31]. Recently, Chen et al. [32] and Rehman and Aydi [33] established some coupled FP and common FP results, respectively, in FCMs with integral types of applications. Waheed et al. [34] proved some coupled FP theorems in FCMSs depending on another function with an application to Volterra integral equations.

In this paper, we prove some rational-type unique coupled FP theorems in FCMSs under the rational type $f c$-contractive conditions with supportive examples. In addition, to verify the validity of our work, we present an application of a Lebesgue integral-type contraction condition theorem to support our work. The layout of this paper is as follows: Section 2 consists of some basic preliminary concepts. In Section 3, we define the rational coupled $f c$-contractive mapping in FCMS and prove some unique rational coupled FP results in complete FCMSs with suitable examples. Section 4 deals with the application of Lebesgue integral-type contraction mapping to get the existence result of unique coupled FP theorems in complete FCMSs.

\section{Preliminaries}

In this section, we recall some basic definitions and lemmas related to our main results. Throughout the complete paper, $\mathbb{N}$ represents a set of natural numbers and $\mathrm{T}$-norm represents a continuous $t$-norm as defined in [35].

Definition 1. An operation $*:[0,1]^{2} \longrightarrow[0,1]$ would be a $\mathrm{T}$-norm if $*$ fulfils the following conditions:

(1) $*$ is associative, commutative, and continuous

(2) $1 * \kappa_{1}=\kappa_{1}, \forall \kappa_{1} \in[0,1]$

(3) $\kappa_{1} * \kappa_{2} \leq \kappa_{3} * \kappa_{4}$ whenever $\kappa_{1} \leq \kappa_{3}$ and $\kappa_{2} \leq \kappa_{4}$, for $\kappa_{1}, \kappa_{2}, \kappa_{3}, \kappa_{4} \in[0,1]$

Definition 2. Let $E$ be a real Banach space, $0 \in E$. Then, a subset $\mathbf{C} \subset E$ is called a cone:

(1) If $\mathbf{C} \neq \varnothing$, closed, and $\mathbf{C} \neq\{0\}$

(2) If $\kappa_{1}, \kappa_{2} \geq 0$ and $g, z \in \mathbf{C}$, then $\kappa_{1} g+\kappa_{2} z \in \mathbf{C}$

(3) If $-z, z \in \mathbf{C}$, then $z=0$.

A partial ordering is defined on a given cone $\mathrm{C} \subset \mathrm{E}$ by $g \leq z \Leftrightarrow z-g \in$ C. $g<z$ stands for $g \leq z$ and $g \neq z$, while $g \ll z$ stands for $z-g \in \operatorname{int}(\mathbf{C})$. In this paper, all cones have a nonempty interior.
Definition 3. A 3-tuple $\left(G, M_{r}, *\right)$ is said to be a FMS if $G$ is any set, $*$ is a $\mathrm{T}$-norm, and $M_{r}$ is a fuzzy set on $G \times G \times$ $(0, \infty)$ which satisfies the following:

(1) $M_{r}\left(g_{1}, g_{2}, T\right)>0$

(2) $M_{r}\left(g_{1}, g_{2}, T\right)=0 \Leftrightarrow g_{1}=g_{2}$

(3) $M_{r}\left(g_{1}, g_{2}, T\right)=M_{r}\left(g_{2}, g_{1}, T\right)$

(4) $M_{r}\left(g_{1}, g_{3}, \mathrm{~T}\right) * M_{r}\left(g_{3}, g_{2}, s\right) \leq M_{r}\left(g_{1}, g_{2}, \mathrm{~T}+s\right)$

(5) $M_{r}\left(g_{1}, g_{2},.\right):(0, \infty) \longrightarrow[0,1]$ is continuous; $\forall g_{1}, g_{2}, g_{3} \in G$ and $s, T>0$

Definition 4. A 3-tuple ( $\left.G, M_{r}, *\right)$ is said to be a FCMS if $\mathbf{C}$ is a cone of $E, G$ is an arbitrary set, $*$ is a $\mathrm{T}$-norm, and $M_{r}$ is a fuzzy set on $G \times G \times \operatorname{int}(\mathbf{C})$ which satisfies the following:

(1) $M_{r}\left(g_{1}, g_{2}, T\right)>0$

(2) $M_{r}\left(g_{1}, g_{2}, T\right)=0 \Leftrightarrow g_{1}=g_{2}$

(3) $M_{r}\left(g_{1}, g_{2}, T\right)=M_{r}\left(g_{2}, g_{1}, T\right)$

(4) $M_{r}\left(g_{1}, g_{3}, T\right) * M_{r}\left(g_{3}, g_{2}, s\right) \leq M_{r}\left(g_{1}, g_{2}, T+s\right)$

(5) $M_{r}\left(g_{1}, g_{2},.\right): \operatorname{int}(C) \longrightarrow[0,1]$ is continuous; $\forall g_{1}, g_{2}, g_{3} \in G$, and $s, \top \gg 0$

Definition 5. Let a 3-tuple $\left(G, M_{r}, *\right)$ be a FCMS and $\varrho \in G$ and a sequence $\left\{g_{J}\right\}$ in $G$

(1) Converges to $\varrho$ if $\gamma \in(0,1)$ and $T \gg 0$ and there is $J_{1} \in \mathbb{N} \ni M_{r}\left(g_{J}, \varrho, T\right)>1-\gamma$, for $J \geq J_{1}$. We may write this $\lim _{J \longrightarrow \infty} g_{J}=\varrho$ or $g_{J} \longrightarrow \varrho$ as $J \longrightarrow \infty$.

(2) Is a Cauchy sequence if $\gamma \in(0,1)$ and $\mathrm{T} \gg 0$ and there is $J_{1} \in \mathbb{N} \ni M_{r}\left(g_{J}, g_{\ell}, T\right)>1-\gamma$, for $J, \ell \geq J_{1}$.

(3) $\left(G, M_{r}, *\right)$ is complete if every Cauchy sequence is convergent in $G$.

(4) Is $f c$-contractive if $\exists \eta \in(0,1)$, satisfying $\left(1 / M_{r}\left(g_{J}, g_{J+1}, T\right)\right)-1 \leq \eta\left(1 / M_{r}\left(g_{J-1}, g_{J}, T\right)-\right.$ $1)$, for $\mathrm{T} \gg 0, J \geq 1$.

Lemma 1. Let $\left(G, M_{r}, *\right)$ be a FCMS, and let a sequence $\left\{g_{J}\right\}$ in $G$ converge to a point $\varrho \in G$ if $M_{r}\left(g_{J}, \varrho, T\right) \longrightarrow 1$ as $J \longrightarrow \infty$, for $\mathrm{T} \gg 0$.

Definition 6. Let $\left(G, M_{r}, *\right)$ be a FCMS. The FCM $M_{r}$ is triangular, if $\left(1 / M_{r}(g, h, T)\right)-1 \leq\left(\left(1 / M_{r}(g, z, T)\right)-\right.$ $1)+\left(\left(1 / M_{r}(z, h, T)\right)-1\right), \forall g, h, z \in G, \top \gg 0 . \Gamma$.

Definition 7 (see [26]). Let $\left(G, M_{r}, *\right)$ be a FCMS and $\Gamma: G \longrightarrow G$. Then, $\Gamma$ is known as a $f c$-contractive if $\exists \eta \in(0,1)$ such that

$$
\frac{1}{M_{r}(\Gamma g, \Gamma h, \mathrm{~T})}-1 \leq \eta\left(\frac{1}{M_{r}(g, h, \mathrm{~T})}-1\right), \quad \forall g, h \in G, \mathrm{~T} \gg 0 .
$$

Definition 8. An element $(g, h) \in G \times G$ is known as a coupled FP of a function $\ddot{F}: G \times G \longrightarrow G$ if 


$$
\Gamma(g, h)=g, \quad \text { and } \Gamma(h, g)=h .
$$

Furthermore, we shall study some unique coupled FP results in FCMSs under the rational coupled $f c$ - contraction conditions with examples. Also, we present an application of the Lebesgue integral-type rational coupled $f c$ - contraction mapping to get a unique rational coupled FP result in FCMSs.

\section{Main Results}

In this section, we shall present our main results with illustrative examples.

Definition 9. Let $\left(G, M_{r}, *\right)$ be a FCMS. A mapping $\Gamma: G \times$ $G \longrightarrow G$ is called a rational coupled $f c-$ contraction if $\exists \eta_{1} \in(0,1)$ and $\eta_{2} \geq 0$ such tha

$$
\begin{aligned}
\frac{1}{M_{r}(\Gamma(g, h), \Gamma(\xi, \kappa), \mathrm{T})}-1 \leq & \eta_{1}\left(\frac{1}{M_{r}(g, \xi, \mathrm{T})}-1\right) \\
& +\eta_{2}\left(\frac{M_{r}(g, \xi, \mathrm{T})}{M_{r}(g, \Gamma(g, h), \mathrm{T}) * M_{r}(\xi, \Gamma(g, h), 2 \mathrm{~T})}-1\right), \quad \forall g, h, \xi, \kappa \in G \text { and } \mathrm{T} \gg 0 .
\end{aligned}
$$

Theorem 1. Assume that $\left(G, M_{r}, *\right)$ be a complete FCMS in which $M_{r}$ is triangular and a mapping $\Gamma: G \times G \longrightarrow G$ is a rational coupled $f c-$ contraction satisfying (3). Then, $\Gamma$ has a unique coupled FP in G.
Proof. Let any $g_{0}, h_{0} \in G$; we define sequences $\left\{g_{J}\right\}$ and $\left\{h_{J}\right\}$ in $G$ such that

$$
\Gamma\left(g_{J}, h_{J}\right)=g_{J+1}, \quad \text { and } \Gamma\left(h_{J}, g_{J}\right)=h_{J+1}, \text { for } J \geq 0 \text {. }
$$

Now, from (3) and (4), for $\mathrm{T} \gg 0$,

$$
\begin{aligned}
\frac{1}{M_{r}\left(g_{J}, g_{J+1}, \mathrm{~T}\right)}-1 & =\frac{1}{M_{r}\left(\Gamma\left(g_{J-1}, h_{J-1}\right), \Gamma\left(g_{J}, h_{J}\right), t\right)}-1 \\
& \leq \eta_{1}\left(\frac{1}{M_{r}\left(g_{J-1}, g_{J}, \mathrm{~T}\right)}-1\right)+\eta_{2}\left(\frac{M_{r}\left(g_{J-1}, g_{J}, \mathrm{~T}\right)}{M_{r}\left(g_{J-1}, \Gamma\left(g_{J-1}, h_{J-1}\right), \mathrm{T}\right) * M_{r}\left(g_{J}, \Gamma\left(g_{J-1}, h_{J-1}\right), 2_{\mathrm{T}}\right)}-1\right) \\
& =\eta_{1}\left(\frac{1}{M_{r}\left(g_{J-1}, g_{J}, \mathrm{~T}\right)}-1\right)+\eta_{2}\left(\frac{M_{r}\left(g_{J-1}, g_{J}, \mathrm{~T}\right)}{M_{r}\left(g_{J-1}, g_{J}, \mathrm{~T}\right) * M_{r}\left(g_{J}, g_{J}, 2_{\mathrm{T}}\right)}-1\right) .
\end{aligned}
$$

This implies that

$$
\frac{1}{M_{r}\left(g_{J}, g_{J+1}, \mathrm{~T}\right)}-1 \leq \eta_{1}\left(\frac{1}{M_{r}\left(g_{J-1}, g_{J}, \mathrm{~T}\right)}-1\right), \quad \text { for } \mathrm{T} \gg 0
$$

Similarly,

$$
\frac{1}{M_{r}\left(g_{J-1}, g_{J}, \mathrm{~T}\right)}-1 \leq \eta_{1}\left(\frac{1}{M_{r}\left(g_{J-2}, g_{J-1}, \mathrm{~T}\right)}-1\right), \quad \text { for } \mathrm{T} \gg 0
$$

Now, from (6) and (7) and by induction, for $\mathrm{T} \gg 0$, we have that

$$
\begin{aligned}
\frac{1}{M_{r}\left(g_{J}, g_{J+1}, \mathrm{~T}\right)}-1 & \leq \eta_{1}\left(\frac{1}{M_{r}\left(g_{J-1}, g_{J}, \mathrm{~T}\right)}-1\right), \\
& \leq \eta_{1}^{2}\left(\frac{1}{M_{r}\left(g_{J-2}, g_{J-1}, \mathrm{~T}\right)}-1\right) \\
& \leq \cdots \leq \eta_{1}^{J}\left(\frac{1}{M_{r}\left(g_{0}, g_{1}, \mathrm{~T}\right)}-1\right) \longrightarrow 0, \text { as } J \longrightarrow \infty .
\end{aligned}
$$

This shows $\left\{g_{J}\right\}$ is a $f c$-contractive sequence; therefore,

$$
\lim _{J \longrightarrow \infty} M_{r}\left(g_{J}, g_{J+1}, \mathrm{~T}\right)=1, \quad \text { for } \mathrm{T} \gg 0 .
$$

Now, for $\ell>J$ and for $\mathrm{T} \gg 0$, 


$$
\begin{aligned}
\frac{1}{M_{r}\left(g_{J}, g_{\ell}, \mathrm{T}\right)}-1 & \leq\left(\frac{1}{M_{r}\left(g_{J}, g_{J+1}, \mathrm{~T}\right)}-1\right)+\left(\frac{1}{M_{r}\left(g_{J+1}, g_{J+2}, \mathrm{~T}\right)}-1\right)+\cdots+\left(\frac{1}{M_{r}\left(g_{\ell-1}, g_{\ell}, \mathrm{T}\right)}-1\right) \\
& \leq \eta_{1}^{J}\left(\frac{1}{M_{r}\left(g_{o}, g_{1}, \mathrm{~T}\right)}-1\right)+\eta_{1}^{J+1}\left(\frac{1}{M_{r}\left(g_{0}, g_{1}, \mathrm{~T}\right)}-1\right)+\cdots+\eta_{1}^{\ell-1}\left(\frac{1}{M_{r}\left(g_{0}, g_{1}, \mathrm{~T}\right)}-1\right) \\
& =\left(\eta_{1}^{J}+\eta_{1}^{J+1}+\cdots+\eta_{1}^{\ell-1}\right)\left(\frac{1}{M_{r}\left(g_{0}, g_{1}, \mathrm{~T}\right)}-1\right) \\
& =\frac{\eta_{1}^{J}}{1-\eta_{1}}\left(\frac{1}{M_{r}\left(g_{o}, g_{1}, \mathrm{~T}\right)}-1\right) \longrightarrow 0, \quad \text { as } J \longrightarrow \infty
\end{aligned}
$$

Hence, $\left\{g_{J}\right\}$ is a Cauchy sequence. Since, by the completeness of $\left(G, M_{r}, *\right), \exists g \in G$, so that

$$
\lim _{J \longrightarrow \infty} M_{r}\left(g_{J}, g, \mathrm{~T}\right)=1, \quad \text { for } \mathrm{T} \gg 0 .
$$

Now, for sequence $\left\{h_{J}\right\}$ and from (3) and (4), for $\mathrm{T} \gg 0$,

$$
\begin{aligned}
\frac{1}{M_{r}\left(h_{J}, h_{J+1}, \mathrm{~T}\right)}-1 & =\frac{1}{M_{r}\left(\Gamma\left(h_{J-1}, g_{J-1}\right), \Gamma\left(h_{J}, g_{J}\right), \mathrm{T}\right)}-1 \\
& \leq \eta_{1}\left(\frac{1}{M_{r}\left(h_{J-1}, h_{J}, \mathrm{~T}\right)}-1\right)+\eta_{2}\left(\frac{M_{r}\left(h_{J-1}, h_{J}, \mathrm{~T}\right)}{M_{r}\left(h_{J-1}, \Gamma\left(h_{J-1}, g_{J-1}\right), \mathrm{T}\right) * M_{r}\left(h_{J}, \Gamma\left(h_{J-1}, g_{J-1}\right), 2_{\mathrm{T}}\right)}-1\right) \\
& =\eta_{1}\left(\frac{1}{M_{r}\left(h_{J-1}, h_{J}, \mathrm{~T}\right)}-1\right)+\eta_{2}\left(\frac{M_{r}\left(h_{J-1}, h_{J}, \mathrm{~T}\right)}{M_{r}\left(h_{J-1}, h_{J}, \mathrm{~T}\right) * M_{r}\left(h_{J}, h_{J}, 2 \mathrm{~T}\right)}-1\right)
\end{aligned}
$$

This implies that

$$
\frac{1}{M_{r}\left(h_{J}, h_{J+1}, \mathrm{~T}\right)}-1 \leq \eta_{1}\left(\frac{1}{M_{r}\left(h_{J-1}, h_{J}, \mathrm{~T}\right)}-1\right), \quad \text { for } \mathrm{T} \gg 0 \text {. }
$$$$
\frac{1}{M_{r}\left(h_{J-1}, h_{J}, \mathrm{~T}\right)}-1 \leq \eta_{1}\left(\frac{1}{M_{r}\left(h_{J-2}, h_{J-1}, \mathrm{~T}\right)}-1\right), \quad \text { for } \mathrm{T} \gg 0 .
$$

Now, from (13) and (14) and by induction, for $\mathrm{T} \gg 0$, we have that

Similarly,

$$
\begin{aligned}
\frac{1}{M_{r}\left(h_{J}, h_{J+1}, \mathrm{~T}\right)}-1 & \leq \eta_{1}\left(\frac{1}{M_{r}\left(h_{J-1}, h_{J}, \mathrm{~T}\right)}-1\right) \\
& \leq \eta_{1}^{2}\left(\frac{1}{M_{r}\left(h_{J-2}, h_{J-1}, \mathrm{~T}\right)}-1\right) \\
& \leq \cdots \leq \eta_{1}^{J}\left(\frac{1}{M_{r}\left(h_{0}, h_{1}, \mathrm{~T}\right)}-1\right) \rightarrow 0, \quad \text { as } J \longrightarrow \infty
\end{aligned}
$$

This shows $\left\{h_{J}\right\}$ is a $f c$-contractive sequence; therefore, 


$$
\lim _{J \longrightarrow \infty} M_{r}\left(h_{J}, h_{J+1}, \mathrm{~T}\right)=1, \quad \text { for } \mathrm{T} \gg 0 .
$$

Now, for $\ell>J$ and for $\mathrm{T} \gg 0$, we have

$$
\begin{aligned}
\frac{1}{M_{r}\left(h_{J}, h_{\ell}, \mathrm{T}\right)}-1 & \leq\left(\frac{1}{M_{r}\left(h_{J}, h_{J+1}, \mathrm{~T}\right)}-1\right)+\left(\frac{1}{M_{r}\left(h_{J+1}, h_{J+2}, \mathrm{~T}\right)}-1\right)+\cdots+\left(\frac{1}{M_{r}\left(h_{\ell-1}, h_{\ell}, \mathrm{T}\right)}-1\right) \\
& \leq \eta_{1}^{J}\left(\frac{1}{M_{r}\left(h_{0}, h_{1}, \mathrm{~T}\right)}-1\right)+\eta_{1}^{J+1}\left(\frac{1}{M_{r}\left(h_{0}, h_{1}, \mathrm{~T}\right)}-1\right)+\cdots+\eta_{1}^{\ell-1}\left(\frac{1}{M_{r}\left(h_{0}, h_{1}, \mathrm{~T}\right)}-1\right) \\
& =\left(\eta_{1}^{J}+\eta_{1}^{J+1}+\cdots+\eta_{1}^{\ell-1}\right)\left(\frac{1}{M_{r}\left(h_{0}, h_{1}, \mathrm{~T}\right)}-1\right) \\
& =\frac{\eta_{1}^{J}}{1-\eta_{1}}\left(\frac{1}{M_{r}\left(h_{0}, h_{1}, \mathrm{~T}\right)}-1\right) \longrightarrow 0, \quad \text { as } J \longrightarrow \infty .
\end{aligned}
$$

Hence, $\left\{h_{J}\right\}$ is a Cauchy sequence. Since, by the completeness of ( $\left.G, M_{r}, *\right), \exists g \in G$ so that

$$
\lim _{J \longrightarrow \infty} M_{r}\left(h_{J}, h, \mathrm{~T}\right)=1, \quad \text { for } \mathrm{T} \gg 0 .
$$

$$
\begin{aligned}
\frac{1}{M_{r}(g, \Gamma(g, h), \mathrm{T})}-1 \leq & \left(\frac{1}{M_{r}\left(g, \Gamma\left(g_{J}, h_{J}\right), \mathrm{T}\right)}-1\right)+\left(\frac{1}{M_{r}\left(\Gamma\left(g_{J}, h_{J}\right), \Gamma(g, h), \mathrm{T}\right)}-1\right), \\
& \leq\left(\frac{1}{M_{r}\left(g, \Gamma\left(g_{J}, h_{J}\right), \mathrm{T}\right)}-1\right)+\eta_{1}\left(\frac{1}{M_{r}\left(g_{\ell}, g, \mathrm{~T}\right)}-1\right)+\eta_{2}\left(\frac{M_{r}\left(g_{J}, g, \mathrm{~T}\right)}{M_{r}\left(g_{J}, \Gamma\left(g_{J}, h_{J}\right), \mathrm{T}\right) * M_{r}\left(g, \Gamma\left(g_{J}, h_{J}\right), 2 \mathrm{~T}\right)}-1\right) \\
& =\left(\frac{1}{M_{r}\left(g, g_{J+1}, \mathrm{~T}\right)}-1\right)+\eta_{1}\left(\frac{1}{M_{r}\left(g_{J}, g, \mathrm{~T}\right)}-1\right) \\
& +\eta_{2}\left(\frac{M_{r}\left(g_{J}, g, \mathrm{~T}\right)}{M_{r}\left(g_{J}, g_{J+1}, \mathrm{~T}\right) * M_{r}\left(g, g_{J+1}, 2 \mathrm{~T}\right)}-1\right) \longrightarrow 0, \quad \text { as } J \longrightarrow \infty .
\end{aligned}
$$

Hence, $M_{r}(g, \Gamma(g, h), T)=1 \Rightarrow g=\Gamma(g, h)$ for $T \gg 0$. Next, we have to prove that $h=\Gamma(h, g)$; therefore, by the triangular property of $M_{r}$ and by the view of (3), (16), and (18), for $T \gg 0$,

$$
\begin{aligned}
\frac{1}{M_{r}(h, \Gamma(h, g), \mathrm{T})}-1 \leq & \left(\frac{1}{M_{r}\left(h, \Gamma\left(h_{J}, g_{J}\right), \mathrm{T}\right)}-1\right)+\left(\frac{1}{M_{r}\left(\Gamma\left(h_{J}, g_{J}\right), \Gamma(h, g), \mathrm{T}\right)}-1\right) \\
& \leq\left(\frac{1}{M_{r}\left(h, \Gamma\left(h_{J}, g_{J}\right), \mathrm{T}\right)}-1\right)+\eta_{1}\left(\frac{1}{M_{r}\left(h_{J}, h, \mathrm{~T}\right)}-1\right)+\eta_{2}\left(\frac{M_{r}\left(h_{J}, h, \mathrm{~T}\right)}{M_{r}\left(h_{J}, \Gamma\left(h_{J}, g_{J}\right), \mathrm{T}\right) * M_{r}\left(h, \Gamma\left(h_{J}, g_{J}\right), 2 \mathrm{~T}\right)}-1\right) \\
& =\left(\frac{1}{M_{r}\left(h, h_{J+1}, \mathrm{~T}\right)}-1\right)+\eta_{1}\left(\frac{1}{M_{r}\left(h_{J}, h, \mathrm{~T}\right)}-1\right) \\
& +\eta_{2}\left(\frac{M_{r}\left(h_{J}, h, \mathrm{~T}\right)}{M_{r}\left(h_{J}, h_{J+1}, \mathrm{~T}\right) * M_{r}\left(h, h_{J+1}, 2 \mathrm{~T}\right)}-1\right) \longrightarrow 0, \quad \text { as } J \longrightarrow \infty .
\end{aligned}
$$


Hence, $M_{r}(g, \Gamma(h, g), \mathrm{T})=1 \Rightarrow \Gamma(h, g)=h$, for $\mathrm{T} \gg 0$.

Uniqueness: suppose $\left(g_{1}, h_{1}\right)$ and $\left(h_{1}, g_{1}\right)$ are other coupled fixed-point pairs in $G \times G$ such that $\Gamma\left(g_{1}, h_{1}\right)=g_{1}$ and $\Gamma\left(h_{1}, g_{1}\right)=h_{1}$. Now, from (3) and by using Definition 4 (4), for $T \gg 0$,

$$
\begin{aligned}
\frac{1}{M_{r}\left(g, g_{1}, \mathrm{~T}\right)}-1 & =\frac{1}{M_{r}\left(\Gamma(g, h), \Gamma\left(g_{1}, h_{1}\right), \mathrm{T}\right)}-1 \\
& \leq \eta_{1}\left(\frac{1}{M_{r}\left(g, g_{1}, \mathrm{~T}\right)}-1\right)+\eta_{2}\left(\frac{M_{r}\left(g, g_{1}, \mathrm{~T}\right)}{M_{r}(g, \Gamma(g, h), \mathrm{T}) * M_{r}\left(g_{1}, \Gamma(g, h), 2 \mathrm{~T}\right)}-1\right) \\
& =\eta_{1}\left(\frac{1}{M_{r}\left(g, g_{1}, \mathrm{~T}\right)}-1\right)+\eta_{2}\left(\frac{M_{r}\left(g, g_{1}, \mathrm{~T}\right)}{M_{r}(g, g, \mathrm{~T}) * M_{r}\left(g_{1}, g, 2 \mathrm{~T}\right)}-1\right) \\
& =\eta_{1}\left(\frac{1}{M_{r}\left(g, g_{1}, \mathrm{~T}\right)}-1\right)=\eta_{1}\left(\frac{1}{M_{r}\left(\Gamma(g, h), \Gamma\left(g_{1}, h_{1}\right), \mathrm{T}\right)}-1\right) \\
& \leq \eta_{1}^{2}\left(\frac{1}{M_{r}\left(g, g_{1}, \mathrm{~T}\right)}-1\right) \leq \cdots \leq \eta_{1}^{J}\left(\frac{1}{M_{r}\left(g, g_{1}, \mathrm{~T}\right)}-1\right) \longrightarrow 0, \quad \text { as } J \longrightarrow \infty
\end{aligned}
$$

Hence, we get that $M_{r}\left(g, g_{1}, T\right)=1 \Rightarrow g=g_{1}$ for $T \gg 0$.

Similarly, again from (3) and by using Definition 4 (4), for $T \gg 0$,

$$
\begin{aligned}
& \frac{1}{M_{r}\left(h, h_{1}, \mathrm{~T}\right)}-1=\frac{1}{M_{r}\left(\Gamma(h, g), \Gamma\left(h_{1}, g_{1}\right), \mathrm{T}\right)}-1, \\
& \leq \eta_{1}\left(\frac{1}{M_{r}\left(h, h_{1}, \mathrm{~T}\right)}-1\right)+\eta_{2}\left(\frac{M_{r}\left(h, h_{1}, \mathrm{~T}\right)}{M_{r}(h, \Gamma(h, g), \mathrm{T}) * M_{r}\left(h_{1}, \Gamma(h, g), 2 \mathrm{~T}\right)}-1\right) \\
&=\eta_{1}\left(\frac{1}{M_{r}\left(h, h_{1}, \mathrm{~T}\right)}-1\right)+\eta_{2}\left(\frac{M_{r}\left(h, h_{1}, \mathrm{~T}\right)}{M_{r}\left(h, h_{1}, \mathrm{~T}\right) * M_{r}\left(h_{1}, h, 2 \mathrm{~T}\right)}-1\right) \\
&=\eta_{1}\left(\frac{1}{M_{r}\left(h, h_{1}, \mathrm{~T}\right)}-1\right)=\eta_{1}\left(\frac{1}{M_{r}\left(\Gamma(h, g), \Gamma\left(h_{1}, g_{1}\right), \mathrm{T}\right)}-1\right) \\
& \leq \eta_{1}^{2}\left(\frac{1}{M_{r}\left(h, h_{1}, \mathrm{~T}\right)}-1\right) \leq \cdots \leq \eta_{1}^{J}\left(\frac{1}{M_{r}\left(h, h_{1}, \mathrm{~T}\right)}-1\right) \longrightarrow 0, \quad \text { as } J \longrightarrow \infty . \\
& \text { get that } \quad M_{r}\left(h, h_{1}, \mathrm{~T}\right)=1 \Rightarrow h=h_{1} \quad \text { for } \quad \forall \quad M_{r}(g, h, \mathrm{~T})=\frac{\mathrm{T}}{\mathrm{T}+|g-h|}, \quad \forall g, h \in G \text { and } \mathrm{T}>0 .
\end{aligned}
$$
$\mathrm{T} \gg 0$.

Hence, we get that $M_{r}\left(h, h_{1}, \mathrm{~T}\right)=1 \Rightarrow h=h_{1}$ for

Corollary 1. Let $\Gamma: G \times G \longrightarrow G$ be a mapping on a complete FCMS $\left(G, M_{r}, *\right)$ in which $M_{r}$ is triangular and $\Gamma$ satisfies the inequality

$$
\frac{1}{M_{r}(\Gamma(g, h), \Gamma(\xi, \kappa), \mathrm{T})}-1 \leq \eta_{1}\left(\frac{1}{M_{r}(g, \xi, \mathrm{T})}-1\right),
$$

$\forall g, h, \xi, \kappa \in G, T \gg 0$, and $\eta_{1} \in(0,1)$. Then, $\Gamma$ has a unique coupled FP in G.

Example 1. Let $G=[0, \infty) ; *$ is a $\mathrm{T}$-norm, and $M_{r}: G \times G \times$ $(0, \infty) \longrightarrow[0,1]$ is defined as
Then, one can easily prove the triangular property of FCM from the above example and $\left(G, M_{r}, *\right)$ is a complete FCMS. We define a mapping $\Gamma: G \times G \longrightarrow G$ by

$$
\Gamma(g, h)= \begin{cases}\frac{3 g}{8}, & g, h \in[0,1], \\ \frac{g+5 h}{4}-\frac{7}{6}, & g, h \in[1, \infty) .\end{cases}
$$

Then, we have 


$$
\begin{aligned}
\frac{1}{M_{r}(\Gamma(g, h), \Gamma(\xi, \kappa), \mathrm{T})}-1 & =\frac{1}{M_{r}((3 g / 8),(3 \xi / 8), \mathrm{T})}-1 \\
& =\frac{3}{8 \mathrm{~T}}|g-\xi| \\
& =\frac{3}{8}\left(\frac{1}{M_{r}(g, \xi, \mathrm{T})}-1\right) .
\end{aligned}
$$

$\forall g, h, \xi, \kappa \in G$ and $\mathrm{T} \gg 0$. Hence, we proved that $\Gamma: G \times G \longrightarrow G$ is a rational coupled $f c-$ contractive. Now, by using Definition 4 (4), for $\mathrm{T} \gg 0$,

$$
\begin{aligned}
\frac{M_{r}(g, \xi, \mathrm{T})}{M_{r}(g, \Gamma(g, h), \mathrm{T}) * M_{r}(\xi, \Gamma(g, h), 2 \mathrm{~T})}-1 & \leq \frac{M_{r}(g, \xi, \mathrm{T})}{M_{r}(g, \Gamma(g, h), \mathrm{T}) * M_{r}(\xi, g, \mathrm{~T}) * M_{r}(g, \Gamma(g, h), \mathrm{T})}-1, \\
& =\frac{1}{M_{r}(g, \Gamma(g, h), \mathrm{T}) * M_{r}(g, \Gamma(g, h), \mathrm{T})}-1 \\
& =\frac{1}{\left(M_{r}(g, \Gamma(g, h), \mathrm{T})\right)^{2}}-1=\frac{5 g}{64 \mathrm{~T}^{2}}(5 g+16 \mathrm{~T}) .
\end{aligned}
$$

$\forall g, h, \xi, \kappa \in G$. Hence, from the above, we conclude that all the conditions of Theorem 1 are satisfied with $\eta_{1}=3 / 8$, $\eta_{2} \in[0,8 / 13]$, and $\Gamma(g, h)=\Gamma(7 / 3,7 / 3)=7 / 3 \in[0, \infty)$.
Theorem 2. Let $\Gamma: G \times G \longrightarrow G$ be a mapping on a complete FCMS $\left(G, M_{r}\right.$, *) in which $M_{r}$ is triangular and $\Gamma$ satisfies the inequality

$$
\begin{aligned}
\frac{1}{M_{r}(\Gamma(g, h), \Gamma(\xi, \kappa), \mathrm{T})}-1 \leq & \eta_{1}\left(\frac{1}{M_{r}(g, \xi, \mathrm{T})}-1\right)+\eta_{2}\left(\frac{M_{r}(g, \xi, \mathrm{T}) * M_{r}(\xi, \Gamma(\xi, \kappa), \mathrm{T})}{M_{r}(g, \Gamma(g, h), \mathrm{T}) * M_{r}(g, \Gamma(\xi, \kappa), 2 \mathrm{~T})}-1\right) \\
& +\eta_{3}\left(\frac{M_{r}(g, \Gamma(g, h), \mathrm{T})}{M_{r}\left(g, \Gamma(\xi, \kappa), 2_{\mathrm{T}}\right)}-1+\frac{M_{r}(\xi, \Gamma(\xi, \kappa), \mathrm{T})}{M_{r}(g, \Gamma(\xi, \kappa), 2 \mathrm{~T})}-1\right) \\
& +\eta_{4}\left(\frac{1}{M_{r}(g, \Gamma(g, h), \mathrm{T})}-1+\frac{1}{M_{r}(\xi, \Gamma(\xi, \kappa), \mathrm{T})}-1\right)
\end{aligned}
$$

$\forall g, h, \xi, \kappa \in G, \quad \mathrm{~T} \gg 0, \quad \eta_{1} \in(0,1)$, and $\eta_{2}, \eta_{3}, \eta_{4} \geq 0$ with $\Gamma\left(g_{J}, h_{J}\right)=g_{J+1}, \quad$ and $\Gamma\left(h_{J}, g_{J}\right)=h_{J+1}$, for $J \geq 0$. $\left(\eta_{1}+\eta_{2}+2 \eta_{3}+2 \eta_{4}\right)<1$. Then, $\Gamma$ has a unique coupled FP in $G$.

Now, from (28) and (29), for $\mathrm{T} \gg 0$,

Proof. Let any $g_{0}, h_{0} \in G$; we define sequences $\left\{g_{J}\right\}$ and $\left\{h_{J}\right\}$ in $G$ such that

$$
\begin{aligned}
\frac{1}{M_{r}\left(g_{J}, g_{J+1}, \mathrm{~T}\right)}-1= & \frac{1}{M_{r}\left(\Gamma\left(g_{J-1}, h_{J-1}\right), \Gamma\left(g_{J}, h_{J}\right), \mathrm{T}\right)}-1, \\
\leq & \eta_{1}\left(\frac{1}{M_{r}\left(g_{J-1}, g_{J}, \mathrm{~T}\right)}-1\right)+\eta_{2}\left(\frac{M_{r}\left(g_{J-1}, g_{J}, \mathrm{~T}\right) * M_{r}\left(g_{J}, \Gamma\left(g_{J}, h_{J}\right), \mathrm{T}\right)}{M_{r}\left(g_{J-1}, \Gamma\left(g_{J-1}, h_{J-1}\right), \mathrm{T}\right) * M_{r}\left(g_{J-1}, \Gamma\left(g_{J}, h_{J}\right), 2 \mathrm{~T}\right)}-1\right) \\
& +\eta_{3}\left(\frac{M_{r}\left(g_{J-1}, \Gamma\left(g_{J-1}, h_{J-1}\right), \mathrm{T}\right)}{M_{r}\left(g_{J-1}, \Gamma\left(g_{J}, h_{J}\right), 2 \mathrm{~T}\right)}-1+\frac{M_{r}\left(g_{J}, \Gamma\left(g_{J}, h_{J}\right), \mathrm{T}\right)}{M_{r}\left(g_{J-1}, \Gamma\left(g_{J}, h_{J}\right), 2 \mathrm{~T}\right)}-1\right)
\end{aligned}
$$




$$
\begin{aligned}
& +\eta_{4}\left(\frac{1}{M_{r}\left(g_{J-1}, \Gamma\left(g_{J-1}, h_{J-1}\right), \mathrm{T}\right)}-1+\frac{1}{M_{r}\left(g_{J}, \Gamma\left(g_{J}, h_{J}\right), \mathrm{T}\right)}-1\right) \\
= & \eta_{1}\left(\frac{1}{M_{r}\left(g_{J-1}, g_{J}, \mathrm{~T}\right)}-1\right)+\eta_{2}\left(\frac{M_{r}\left(g_{J-1}, g_{J}, \mathrm{~T}\right) * M_{r}\left(g_{J}, g_{J+1}, \mathrm{~T}\right)}{M_{r}\left(g_{J-1}, g_{J}, \mathrm{~T}\right) * M_{r}\left(g_{J-1}, g_{J+1}, 2 \mathrm{~T}\right)}-1\right) \\
& +\eta_{3}\left(\frac{M_{r}\left(g_{J-1}, g_{J}, \mathrm{~T}\right)}{M_{r}\left(g_{J-1}, g_{J+1}, 2 \mathrm{~T}\right)}-1+\frac{M_{r}\left(g_{J}, g_{J+1}, \mathrm{~T}\right)}{M_{r}\left(g_{J-1}, g_{J+1}, 2 \mathrm{~T}\right)}-1\right)+\eta_{4}\left(\frac{1}{M_{r}\left(g_{J-1}, g_{J}, \mathrm{~T}\right)}-1+\frac{1}{M_{r}\left(g_{J}, g_{J+1}, \mathrm{~T}\right)}-1\right) .
\end{aligned}
$$

Now, by Definition 4 (4), for $\mathrm{T} \gg 0$,

$$
\begin{aligned}
\frac{1}{M_{r}\left(g_{J}, g_{J+1}, \mathrm{~T}\right)}-1 \leq & \eta_{1}\left(\frac{1}{M_{r}\left(g_{J-1}, g_{J}, \mathrm{~T}\right)}-1\right)+\eta_{2}\left(\frac{M_{r}\left(g_{J-1}, g_{J}, \mathrm{~T}\right) * M_{r}\left(g_{J}, g_{J+1}, \mathrm{~T}\right)}{M_{r}\left(g_{J-1}, g_{J}, \mathrm{~T}\right) * M_{r}\left(g_{J-1}, g_{J}, \mathrm{~T}\right) * M_{r}\left(g_{J}, g_{J+1}, \mathrm{~T}\right)}-1\right) \\
& +\eta_{3}\left(\frac{M_{r}\left(g_{J-1}, g_{J}, \mathrm{~T}\right)}{M_{r}\left(g_{J-1}, g_{J}, \mathrm{~T}\right) * M_{r}\left(g_{J}, g_{J+1}, \mathrm{~T}\right)}-1+\frac{M_{r}\left(g_{J}, g_{J+1}, \mathrm{~T}\right)}{M_{r}\left(g_{J-1}, g_{J}, \mathrm{~T}\right) * M_{r}\left(g_{J}, g_{J+1}, \mathrm{~T}\right)}-1\right) \\
& +\eta_{4}\left(\frac{1}{M_{r}\left(g_{J-1}, g_{J}, \mathrm{~T}\right)}-1+\frac{1}{M_{r}\left(g_{J}, g_{J+1}, \mathrm{~T}\right)}-1\right) .
\end{aligned}
$$

After simplification, we get that

$$
\frac{1}{M_{r}\left(g_{J}, g_{J+1}, \mathrm{~T}\right)}-1 \leq \rho\left(\frac{1}{M_{r}\left(g_{J-1}, g_{J}, \mathrm{~T}\right)}-1\right), \quad \text { for } \mathrm{T} \gg 0 \text {. }
$$

where $\rho=\left(\eta_{1}+\eta_{2}+\eta_{3}+\eta_{4}\right) /\left(1-\eta_{3}-\eta_{4}\right)<1$. Similarly, again, by using (28) and Definition $4(4)$, we get that

$$
\frac{1}{M_{r}\left(g_{J-1}, g_{J}, \mathrm{~T}\right)}-1 \leq \rho\left(\frac{1}{M_{r}\left(g_{J-2}, g_{J-1}, \mathrm{~T}\right)}-1\right), \quad \text { for } \mathrm{T} \gg 0 .
$$

where $\rho=\left(\eta_{1}+\eta_{2}+\eta_{3}+\eta_{4}\right) /\left(1-\eta_{3}-\eta_{4}\right)<1$. Now, from (32) and (33) and by induction, for $\mathrm{T} \gg 0$, we have that

$$
\begin{aligned}
\frac{1}{M_{r}\left(g_{J}, g_{J+1}, \mathrm{~T}\right)}-1 & \leq \rho\left(\frac{1}{M_{r}\left(g_{J-1}, g_{J}, \mathrm{~T}\right)}-1\right), \\
& \leq \rho^{2}\left(\frac{1}{M_{r}\left(g_{J-2}, g_{J-1}, \mathrm{~T}\right)}-1\right) \\
& \leq \cdots \leq \rho^{J}\left(\frac{1}{M_{r}\left(g_{0}, g_{1}, \mathrm{~T}\right)}-1\right) \longrightarrow 0, \quad \text { as } J \longrightarrow \infty
\end{aligned}
$$

This shows $\left\{g_{J}\right\}$ is a $f c$-contractive sequence; therefore, 


$$
\lim _{\ell \longrightarrow \infty} M_{r}\left(g_{J}, g_{J+1}, T\right)=1, \quad \text { for } \mathrm{T} \gg 0 .
$$

Now, for $\ell>J$ and for $\mathrm{T} \gg 0$, we have

$$
\begin{aligned}
\frac{1}{M_{r}\left(g_{J}, g_{\ell}, \mathrm{T}\right)}-1 & \leq\left(\frac{1}{M_{r}\left(g_{J}, g_{J+1}, \mathrm{~T}\right)}-1\right)+\left(\frac{1}{M_{r}\left(g_{J+1}, g_{J+2}, \mathrm{~T}\right)}-1\right)+\cdots+\left(\frac{1}{M_{r}\left(g_{\ell-1}, g_{\ell}, \mathrm{T}\right)}-1\right) \\
& \leq \rho^{J}\left(\frac{1}{M_{r}\left(g_{o}, g_{1}, \mathrm{~T}\right)}-1\right)+\rho^{J+1}\left(\frac{1}{M_{r}\left(g_{0}, g_{1}, \mathrm{~T}\right)}-1\right)+\cdots+\rho^{\ell-1}\left(\frac{1}{M_{r}\left(g_{0}, g_{1}, \mathrm{~T}\right)}-1\right) \\
& =\left(\rho^{J}+\rho^{J+1}+\cdots+\rho^{\ell-1}\right)\left(\frac{1}{M_{r}\left(g_{0}, g_{1}, \mathrm{~T}\right)}-1\right) \\
& =\frac{\rho^{J}}{1-\rho}\left(\frac{1}{M_{r}\left(g_{o}, g_{1}, \mathrm{~T}\right)}-1\right) \longrightarrow 0, \quad \text { as } J \longrightarrow \infty
\end{aligned}
$$

Hence, $\left\{g_{J}\right\}$ is a Cauchy sequence. Since, by the completeness of $\left(G, M_{r}, *\right), \exists g \in G$ so that

$$
\lim _{J \longrightarrow \infty} M\left(g_{J}, g, T\right)=1, \quad \text { for } \mathrm{T} \gg 0 .
$$

Now, for sequence $\left\{h_{J}\right\}$, from (28) and (29), for $\mathrm{T} \gg 0$,

$$
\begin{aligned}
\frac{1}{M_{r}\left(h_{J}, h_{J+1}, \mathrm{~T}\right)}-1= & \frac{1}{M_{r}\left(\Gamma\left(h_{J-1}, g_{J-1}\right), \Gamma\left(h_{J}, g_{J}\right), \mathrm{T}\right)}-1 \\
\leq & \eta_{1}\left(\frac{1}{M_{r}\left(h_{J-1}, h_{J}, \mathrm{~T}\right)}-1\right)+\eta_{2}\left(\frac{M_{r}\left(h_{J-1}, h_{J}, \mathrm{~T}\right) * M_{r}\left(h_{J}, \Gamma\left(h_{J}, g_{J}\right), \mathrm{T}\right)}{M_{r}\left(h_{J-1}, \Gamma\left(h_{J-1}, g_{J-1}\right), \mathrm{T}\right) * M_{r}\left(h_{J-1}, \Gamma\left(h_{J}, g_{J}\right), 2 \mathrm{~T}\right)}-1\right) \\
& +\eta_{3}\left(\frac{M_{r}\left(h_{J-1}, \Gamma\left(h_{J-1}, g_{J-1}\right), \mathrm{T}\right)}{M_{r}\left(h_{J-1}, \Gamma\left(h_{J}, g_{J}\right), 2 \mathrm{~T}\right)}-1+\frac{M_{r}\left(h_{J}, \Gamma\left(h_{J}, g_{J}\right), \mathrm{T}\right)}{M_{r}\left(h_{J-1}, \Gamma\left(h_{J}, g_{J}\right), 2 \mathrm{~T}\right)}-1\right) \\
& +\eta_{4}\left(\frac{1}{M_{r}\left(h_{J-1}, \Gamma\left(h_{J-1}, g_{J-1}\right), \mathrm{T}\right)}-1+\frac{1}{M_{r}\left(h_{J}, \Gamma\left(h_{J}, g_{J}\right), \mathrm{T}\right)}-1\right) \\
= & \eta_{1}\left(\frac{1}{M_{r}\left(h_{J-1}, h_{J}, \mathrm{~T}\right)}-1\right)+\eta_{2}\left(\frac{M_{r}\left(h_{J-1}, h_{J}, \mathrm{~T}\right) * M_{r}\left(h_{J}, h_{J+1}, \mathrm{~T}\right)}{M_{r}\left(h_{J-1}, h_{J}, \mathrm{~T}\right) * M_{r}\left(h_{J-1}, h_{J+1}, 2 \mathrm{~T}\right)}-1\right) \\
& +\eta_{3}\left(\frac{M_{r}\left(h_{J-1}, h_{J}, \mathrm{~T}\right)}{M_{r}\left(h_{J-1}, h_{J+1}, 2 \mathrm{~T}\right)}-1+\frac{M_{r}\left(h_{J}, h_{J+1}, \mathrm{~T}\right)}{M_{r}\left(h_{J-1}, h_{J+1}, 2 \mathrm{~T}\right)}-1\right)+\eta_{4}\left(\frac{1}{M_{r}\left(h_{J-1}, h_{J}, \mathrm{~T}\right)}-1+\frac{1}{M_{r}\left(h_{J}, h_{J+1}, \mathrm{~T}\right)}-1\right) .
\end{aligned}
$$

Now, again by Definition 4 (4), for $\mathrm{T} \gg 0$,

$$
\begin{aligned}
\frac{1}{M_{r}\left(h_{J}, h_{J+1}, \mathrm{~T}\right)}-1 \leq & \eta_{1}\left(\frac{1}{M_{r}\left(h_{J-1}, h_{J}, \mathrm{~T}\right)}-1\right)+\eta_{2}\left(\frac{M_{r}\left(h_{J-1}, h_{J}, \mathrm{~T}\right) * M_{r}\left(h_{J}, h_{J+1}, \mathrm{~T}\right)}{M_{r}\left(h_{J-1}, h_{J}, \mathrm{~T}\right) * M_{r}\left(h_{J-1}, h_{J}, \mathrm{~T}\right) * M_{r}\left(h_{J}, h_{J+1}, \mathrm{~T}\right)}-1\right) \\
& +\eta_{3}\left(\frac{M_{r}\left(h_{J-1}, h_{J}, \mathrm{~T}\right)}{M_{r}\left(h_{J-1}, h_{J}, \mathrm{~T}\right) * M_{r}\left(h_{J}, h_{J+1}, \mathrm{~T}\right)}-1+\frac{M_{r}\left(h_{J}, h_{J+1}, \mathrm{~T}\right)}{M_{r}\left(h_{J-1}, h_{J}, \mathrm{~T}\right) * M_{r}\left(h_{J}, h_{J+1}, \mathrm{~T}\right)}-1\right) \\
& +\eta_{4}\left(\frac{1}{M_{r}\left(h_{J-1}, h_{J}, \mathrm{~T}\right)}-1+\frac{1}{M_{r}\left(h_{J}, h_{J+1}, \mathrm{~T}\right)}-1\right) .
\end{aligned}
$$


After simplification, we get that

$$
\frac{1}{M_{r}\left(h_{J}, h_{J+1}, \mathrm{~T}\right)}-1 \leq \rho\left(\frac{1}{M_{r}\left(h_{J-1}, h_{J}, \mathrm{~T}\right)}-1\right), \quad \text { for } \mathrm{T} \gg 0
$$$$
\frac{1}{M_{r}\left(h_{J-1}, h_{J}, \mathrm{~T}\right)}-1 \leq \rho\left(\frac{1}{M_{r}\left(h_{J-2}, h_{J-1}, \mathrm{~T}\right)}-1\right), \quad \text { for } \mathrm{T} \gg 0 .
$$

where the value of $\rho$ is same as in (32). Similarly, again by using (28) and Definition 4 (4), for $\mathrm{T} \gg 0$, we get that

Now, from (40) and (41) and by induction, for $\mathrm{T} \gg 0$, we have that

$$
\begin{aligned}
\frac{1}{M_{r}\left(h_{J}, h_{J+1}, \mathrm{~T}\right)}-1 & \leq \rho\left(\frac{1}{M_{r}\left(h_{J-1}, h_{J}, \mathrm{~T}\right)}-1\right) \\
& \leq \rho^{2}\left(\frac{1}{M_{r}\left(h_{J-2}, h_{J-1}, \mathrm{~T}\right)}-1\right) \\
& \leq \cdots \leq \rho^{J}\left(\frac{1}{M_{r}\left(h_{0}, h_{1}, \mathrm{~T}\right)}-1\right) \rightarrow 0, \quad \text { as } J \longrightarrow \infty
\end{aligned}
$$

This shows $\left\{h_{J}\right\}$ is a $f c$-contractive sequence; therefore,

$$
\lim _{J \longrightarrow \infty} M_{r}\left(h_{J}, h_{J+1}, \mathrm{~T}\right)=1, \quad \text { for } \mathrm{T} \gg 0 .
$$

Now, for $\ell>J$ and for $\mathrm{T} \gg 0$,

$$
\begin{aligned}
\frac{1}{M_{r}\left(h_{J}, h_{J}, \mathrm{~T}\right)}-1 & \leq\left(\frac{1}{M_{r}\left(h_{J}, h_{J+1}, \mathrm{~T}\right)}-1\right)+\left(\frac{1}{M_{r}\left(h_{J+1}, h_{J+2}, \mathrm{~T}\right)}-1\right)+\cdots+\left(\frac{1}{M_{r}\left(h_{\ell-1}, h_{\ell}, \mathrm{T}\right)}-1\right) \\
& \leq \rho^{J}\left(\frac{1}{M_{r}\left(h_{o}, h_{1}, \mathrm{~T}\right)}-1\right)+\rho^{J+1}\left(\frac{1}{M_{r}\left(h_{0}, h_{1}, \mathrm{~T}\right)}-1\right)+\cdots+\rho^{\ell-1}\left(\frac{1}{M_{r}\left(h_{0}, h_{1}, \mathrm{~T}\right)}-1\right) \\
& =\left(\rho^{J}+\rho^{J+1}+\cdots+\rho^{\ell-1}\right)\left(\frac{1}{M_{r}\left(h_{0}, h_{1}, \mathrm{~T}\right)}-1\right) \\
& =\frac{\rho^{J}}{1-\rho}\left(\frac{1}{M_{r}\left(h_{o}, h_{1}, \mathrm{~T}\right)}-1\right) \longrightarrow 0, \quad \text { as } J \longrightarrow \infty .
\end{aligned}
$$

Hence, $\left\{h_{J}\right\}$ is a Cauchy sequence. Since, by the completeness of $\left(G, M_{r}, *\right), \exists h \in G$ so that

$$
\lim _{J \longrightarrow \infty} M_{r}\left(h_{J}, h, \mathrm{~T}\right)=1, \quad \text { for } \mathrm{T} \gg 0 .
$$

Now, we shall prove that $\Gamma(g, h)=g$. Since $M_{r}$ is triangular,

$$
\begin{aligned}
\frac{1}{M_{r}(g, \Gamma(g, h), \mathrm{T})}-1= & \left(\frac{1}{M_{r}\left(g, g_{J+1}, \mathrm{~T}\right)}-1\right) \\
& +\left(\frac{1}{M_{r}\left(g_{J+1}, \Gamma(g, h), t\right)}-1\right), \quad \text { for } \mathrm{T} \gg 0 .
\end{aligned}
$$


Now, by the view of (28), (35), and (37), and by using Definition 4 (4), for $\mathrm{T} \gg 0$,

$$
\begin{aligned}
\frac{1}{M_{r}\left(g_{J+1}, \Gamma(g, h), \mathrm{T}\right)}-1= & \left(\frac{1}{M_{r}\left(\Gamma\left(g_{J}, h_{J}\right), \Gamma(g, h), \mathrm{T}\right)}-1\right) \\
\leq & \eta_{1}\left(\frac{1}{M_{r}\left(g_{J}, g, \mathrm{~T}\right)}-1\right)+\eta_{2}\left(\frac{M_{r}\left(g_{J}, g, \mathrm{~T}\right) * M_{r}(g, \Gamma(g, h), \mathrm{T})}{M_{r}\left(g_{J}, \Gamma\left(g_{J}, h_{J}\right), \mathrm{T}\right) * M_{r}\left(g_{J}, \Gamma(g, h), 2 \mathrm{~T}\right)}-1\right) \\
& +\eta_{3}\left(\frac{M_{r}\left(g_{J}, \Gamma\left(g_{J}, h_{J}\right), \mathrm{T}\right)}{M_{r}\left(g_{J}, \Gamma(g, h), 2 \mathrm{~T}\right)}-1+\frac{M_{r}(g, \Gamma(g, h), \mathrm{T})}{M_{r}\left(g_{J}, \Gamma(g, h), 2 \mathrm{~T}\right)}-1\right) \\
& +\eta_{4}\left(\frac{1}{M_{r}\left(g_{J}, \Gamma\left(g_{J}, h_{J}\right), \mathrm{T}\right)}-1+\frac{1}{M_{r}(g, \Gamma(g, h), \mathrm{T})}-1\right) \\
\leq & \eta_{1}\left(\frac{1}{M_{r}\left(g_{J}, g, \mathrm{~T}\right)}-1\right)+\eta_{2}\left(\frac{M_{r}\left(g_{J}, g, \mathrm{~T}\right) * M_{r}(g, \Gamma(g, h), \mathrm{T})}{M_{r}\left(g_{J}, g_{J+1}, \mathrm{~T}\right) * M_{r}\left(g_{J}, g, \mathrm{~T}\right) * M_{r}(g, \Gamma(g, h), \mathrm{T})}-1\right) \\
& +\eta_{3}\left(\frac{M_{r}\left(g_{J}, g_{J+1}, \mathrm{~T}\right)}{M_{r}\left(g_{J}, g, \mathrm{~T}\right) * M_{r}(g, \Gamma(g, h), \mathrm{T})}-1+\frac{M_{r}(g, \Gamma(g, h), \mathrm{T})}{M_{r}\left(g_{J}, g, \mathrm{~T}\right) * M_{r}(g, \Gamma(g, h), \mathrm{T})}-1\right) \\
& +\eta_{4}\left(\frac{1}{M_{r}\left(g_{J}, g_{J+1}, \mathrm{~T}\right)}-1+\frac{1}{M_{r}(g, \Gamma(g, h), \mathrm{T})}-1\right) \frac{1}{\longrightarrow}\left(\eta_{3}+\eta_{4}\right)\left(\frac{1}{M_{r}(g, \Gamma(g, h), \mathrm{T})}-1\right), \quad \text { as } \longrightarrow \infty .
\end{aligned}
$$

Hence,

Now, from (35), (46), and (48), for $\mathrm{T} \gg 0$,

$$
\begin{aligned}
& \underset{J \longrightarrow \infty}{\limsup }\left(\frac{1}{M_{r}\left(g_{J+1}, \Gamma(g, h), \mathrm{T}\right)}-1\right) \\
& \quad \leq\left(\eta_{3}+\eta_{4}\right)\left(\frac{1}{M_{r}(g, \Gamma(g, h), \mathrm{T})}-1\right), \quad \text { for } \mathrm{T} \gg 0 .
\end{aligned}
$$

$$
\begin{aligned}
& \frac{1}{M_{r}(g, \Gamma(g, h), \mathrm{T})}-1 \leq \\
& \quad\left(\eta_{3}+\eta_{4}\right)\left(\frac{1}{M_{r}(g, \Gamma(g, h), \mathrm{T})}-1\right) \\
& \quad \Rightarrow\left(1-\eta_{3}+\eta_{4}\right)\left(\frac{1}{M_{r}(g, \Gamma(g, h), \mathrm{T})}-1\right) \leq 0, \quad \text { for } \mathrm{T} \gg 0
\end{aligned}
$$

which is a contradiction. As $\left(1-\eta_{3}+\eta_{4}\right) \neq 0$, we get that $M_{r}(g, \Gamma(g, h), \mathrm{T})=1 \Rightarrow \Gamma(g, h)=g$ for $\mathrm{T} \gg 0$. Next, we prove that $\Gamma(h, g)=h$. Now, again from the triangularity of $M$,

$$
\frac{1}{M_{r}(h, \Gamma(h, g), \mathrm{T})}-1 \leq\left(\frac{1}{M_{r}\left(h, h_{J+1}, \mathrm{~T}\right)}-1\right)+\left(\frac{1}{M_{r}\left(h_{J+1}, \Gamma(h, g), \mathrm{T}\right)}-1\right), \quad \text { for } \mathrm{T} \gg 0 .
$$


Now, by the view of (28), (43), and (45), and by using Definition 4 (4), for $\mathrm{T} \gg 0$,

$$
\begin{aligned}
& \frac{1}{M_{r}\left(h_{J+1}, \Gamma(h, g), \mathrm{T}\right)}-1=\left(\frac{1}{M_{r}\left(\Gamma\left(h_{J}, g_{J}\right), \Gamma(h, g), \mathrm{T}\right)}-1\right) \\
& \leq \eta_{1}\left(\frac{1}{M_{r}\left(h_{J}, h, \mathrm{~T}\right)}-1\right)+\eta_{2}\left(\frac{M_{r}\left(h_{J}, h, \mathrm{~T}\right) * M_{r}(h, \Gamma(h, g), \mathrm{T})}{M_{r}\left(h_{J}, \Gamma\left(h_{J}, g_{J}\right), \mathrm{T}\right) * M_{r}\left(h_{J}, \Gamma(h, g), 2 \mathrm{~T}\right)}-1\right) \\
& +\eta_{3}\left(\frac{M_{r}\left(h_{J}, \Gamma\left(h_{J}, g_{J}\right), \mathrm{T}\right)}{M_{r}\left(h_{J}, \Gamma(h, g), 2_{\mathrm{T}}\right)}-1+\frac{M_{r}(h, \Gamma(h, g), \mathrm{T})}{M_{r}\left(h_{J}, \Gamma(h, g), 2 \mathrm{~T}\right)}-1\right) \\
& +\eta_{4}\left(\frac{1}{M_{r}\left(h_{J}, \Gamma\left(h_{J}, g_{J}\right), \mathrm{T}\right)}-1+\frac{1}{M_{r}(h, \Gamma(h, g), \mathrm{T})}-1\right) \\
& \leq \eta_{1}\left(\frac{1}{M_{r}\left(h_{J}, h, \mathrm{~T}\right)}-1\right)+\eta_{2}\left(\frac{M_{r}\left(h_{J}, h, \mathrm{~T}\right) * M_{r}(h, \Gamma(h, g), \mathrm{T})}{M_{r}\left(h_{J}, h_{J+1}, \mathrm{~T}\right) * M_{r}\left(h_{J}, h, \mathrm{~T}\right) * M_{r}(h, \Gamma(h, g), \mathrm{T})}-1\right) \\
& +\eta_{3}\left(\frac{M_{r}\left(h_{J}, h_{J+1}, \mathrm{~T}\right)}{M_{r}\left(h_{J}, h, \mathrm{~T}\right) * M_{r}(h, \Gamma(h, g), \mathrm{T})}-1+\frac{M_{r}(h, \Gamma(h, g), \mathrm{T})}{M_{r}\left(h_{J}, h, \mathrm{~T}\right) * M_{r}(h, \Gamma(h, g), \mathrm{T})}-1\right) \\
& +\eta_{4}\left(\frac{1}{M_{r}\left(h_{J}, h_{J+1}, \mathrm{~T}\right)}-1+\frac{1}{M_{r}(h, \Gamma(h, g), \mathrm{T})}-1\right) \longrightarrow\left(\eta_{3}+\eta_{4}\right)\left(\frac{1}{M_{r}(h, \Gamma(h, g), \mathrm{T})}-1\right), \quad \text { as } J \longrightarrow \infty .
\end{aligned}
$$

Hence,

Now, from (43), (50), and (52), for $\mathrm{T} \gg 0$,

$$
\begin{aligned}
& \underset{J \longrightarrow \infty}{\limsup }\left(\frac{1}{M_{r}\left(h_{J+1}, \Gamma(h, g), \mathrm{T}\right)}-1\right) \\
& \quad \leq\left(\eta_{3}+\eta_{4}\right)\left(\frac{1}{M_{r}(h, \Gamma(h, g), \mathrm{T})}-1\right), \quad \text { for } \mathrm{T} \gg 0 .
\end{aligned}
$$

$$
\begin{aligned}
& \frac{1}{M_{r}(h, \Gamma(h, g), \mathrm{T})}-1 \leq\left(\eta_{3}+\eta_{4}\right)\left(\frac{1}{M_{r}(h, \Gamma(h, g), \mathrm{T})}-1\right) \\
& \Rightarrow\left(1-\eta_{3}+\eta_{4}\right)\left(\frac{1}{M_{r}(h, \Gamma(h, g), \mathrm{T})}-1\right) \leq 0, \quad \text { for } \mathrm{T} \gg 0,
\end{aligned}
$$

which is a contradiction. As $\left(1-\eta_{3}+\eta_{4}\right) \neq 0$, we get that $M_{r}(h, \Gamma(h, g), \mathrm{T})=1 \Rightarrow \Gamma(h, g)=h$ for $\mathrm{T} \gg 0$.
Uniqueness: suppose $\left(g_{1}, h_{1}\right)$ and $\left(h_{1}, g_{1}\right)$ are other coupled FP pairs in $G \times G$ such that $\Gamma\left(g_{1}, h_{1}\right)=g_{1}$ and 
$\Gamma\left(h_{1}, g_{1}\right)=h_{1}$. Now, from (28) and by using Definition $4(4)$, for $T \gg 0$,

$$
\begin{aligned}
\frac{1}{M_{r}\left(g, g_{1}, \mathrm{~T}\right)}-1= & \frac{1}{M_{r}\left(\Gamma(g, h), \Gamma\left(g_{1}, h_{1}\right), \mathrm{T}\right)}-1, \\
\leq & \eta_{1}\left(\frac{1}{M_{r}\left(g, g_{1}, \mathrm{~T}\right)}-1\right)+\eta_{2}\left(\frac{M_{r}\left(g, g_{1}, \mathrm{~T}\right) * M_{r}\left(g_{1}, \Gamma\left(g_{1}, h_{1}\right), \mathrm{T}\right)}{M_{r}(g, \Gamma(g, h), \mathrm{T}) * M_{r}\left(g, \Gamma\left(g_{1}, h_{1}\right), 2 \mathrm{~T}\right)}-1\right) \\
& +\eta_{3}\left(\frac{M_{r}(g, \Gamma(g, h), \mathrm{T})}{M_{r}\left(g, \Gamma\left(g_{1}, h_{1}\right), 2 \mathrm{~T}\right)}-1+\frac{M_{r}\left(g_{1}, \Gamma\left(g_{1}, h_{1}\right), \mathrm{T}\right)}{M_{r}\left(g, \Gamma\left(g_{1}, h_{1}\right), 2 \mathrm{~T}\right)}-1\right) \\
& +\eta_{4}\left(\frac{1}{M_{r}(g, \Gamma(g, h), \mathrm{T})}-1+\frac{1}{M_{r}\left(g_{1}, \Gamma\left(g_{1}, h_{1}\right), \mathrm{T}\right)}-1\right) \\
= & \eta_{1}\left(\frac{1}{M_{r}\left(g, g_{1}, \mathrm{~T}\right)}-1\right)+\eta_{2}\left(\frac{M_{r}\left(g, g_{1}, \mathrm{~T}\right) * M_{r}\left(g_{1}, g_{1}, \mathrm{~T}\right)}{M_{r}(g, g, \mathrm{~T}) * M_{r}\left(g, g_{1}, 2 \mathrm{~T}\right)}-1\right) \\
& +\eta_{3}\left(\frac{M_{r}(g, g, \mathrm{~T})}{M_{r}\left(g, g_{1}, 2 \mathrm{~T}\right)}-1+\frac{M_{r}\left(g_{1}, g_{1}, \mathrm{~T}\right)}{M_{r}\left(g, g_{1}, 2 \mathrm{~T}\right)}-1\right)+\eta_{4}\left(\frac{1}{M_{r}(g, g, \mathrm{~T})}-1+\frac{1}{M_{r}\left(g_{1}, g_{1}, \mathrm{~T}\right)}-1\right) \\
= & \eta_{1}\left(\frac{1}{M_{r}\left(g, g_{1}, \mathrm{~T}\right)}-1\right)+\eta_{2}\left(\frac{M_{r}\left(g, g_{1}, \mathrm{~T}\right)}{M_{r}\left(g, g_{1}, 2 \mathrm{~T}\right)}-1\right)+\eta_{3}\left(\frac{1}{M_{r}\left(g, g_{1}, 2 \mathrm{~T}\right)}-1+\frac{1}{M_{r}\left(g, g_{1}, 2 \mathrm{~T}\right)}-1\right) \\
\leq & \eta_{1}\left(\frac{1}{M_{r}\left(g, g_{1}, \mathrm{~T}\right)}-1\right)+\eta_{2}\left(\frac{M_{r}\left(g, g_{1}, \mathrm{~T}\right)}{M_{r}\left(g, g_{1}, \mathrm{~T}\right) * M_{r}\left(g_{1}, g_{1}, \mathrm{~T}\right)}-1\right) \\
& +\eta_{3}\left(\frac{1}{M_{r}\left(g, g_{1}, \mathrm{~T}\right) * M_{r}\left(g_{1}, g_{1}, \mathrm{~T}\right)}-1+\frac{1}{M_{r}\left(g, g_{1}, \mathrm{~T}\right) * M_{r}\left(g_{1}, g_{1}, \mathrm{~T}\right)}-1\right) \\
= & \left(\eta_{1}+2 \eta_{3}\right)\left(\frac{1}{M_{r}\left(g, g_{1}, \mathrm{~T}\right)}-1\right)=\left(\eta_{1}+2 \eta_{3}\right)\left(\frac{1}{M_{r}\left(\Gamma(g, h), \Gamma\left(g_{1}, h_{1}\right), \mathrm{T}\right)}-1\right) \\
\leq & \left.\eta_{1}+2 \eta_{3}\right)^{2}\left(\frac{1}{M_{r}\left(g, g_{1}, \mathrm{~T}\right)}-1\right) \leq \ldots \leq\left(\eta_{1}+2 \eta_{3}\right)^{J}\left(\frac{1}{M_{r}\left(g, g_{1}, \mathrm{~T}\right)}-1\right) \\
\longrightarrow & \text { as } \longrightarrow
\end{aligned}
$$

Hence, $M_{r}\left(g, g_{1}, T\right)=1 \Rightarrow g=g_{1}$ for $\mathrm{T} \gg 0$. Next, we shall show that $h=h_{1}$, again from (28), and by using Definition 4 (4), for $\mathrm{T} \gg 0$, we have

$$
\begin{aligned}
\frac{1}{M_{r}\left(h, h_{1}, \mathrm{~T}\right)}-1= & \frac{1}{M_{r}\left(\Gamma(h, g), \Gamma\left(h_{1}, g_{1}\right), \mathrm{T}\right)}-1 \\
\leq & \eta_{1}\left(\frac{1}{M_{r}\left(h, h_{1}, \mathrm{~T}\right)}-1\right)+\eta_{2}\left(\frac{M_{r}\left(h, h_{1}, \mathrm{~T}\right) * M_{r}\left(h_{1}, \Gamma\left(h_{1}, g_{1}\right), \mathrm{T}\right)}{M_{r}(h, \Gamma(h, g), \mathrm{T}) * M_{r}\left(h, \Gamma\left(h_{1}, g_{1}\right), 2 \mathrm{~T}\right)}-1\right) \\
& +\eta_{3}\left(\frac{M_{r}(h, \Gamma(h, g), \mathrm{T})}{M_{r}\left(h, \Gamma\left(h_{1}, g_{1}\right), 2 \mathrm{~T}\right)}-1+\frac{M_{r}\left(h_{1}, \Gamma\left(h_{1}, g_{1}\right), \mathrm{T}\right)}{M_{r}\left(h, \Gamma\left(h_{1}, g_{1}\right), 2 \mathrm{~T}\right)}-1\right) \\
& +\eta_{4}\left(\frac{1}{M_{r}(h, \Gamma(h, g), \mathrm{T})}-1+\frac{1}{M_{r}\left(h_{1}, \Gamma\left(h_{1}, g_{1}\right), \mathrm{T}\right)}-1\right)
\end{aligned}
$$




$$
\begin{aligned}
= & \eta_{1}\left(\frac{1}{M_{r}\left(h, h_{1}, \mathrm{~T}\right)}-1\right)+\eta_{2}\left(\frac{M_{r}\left(h, h_{1}, \mathrm{~T}\right) * M_{r}\left(h_{1}, h_{1}, \mathrm{~T}\right)}{M_{r}\left(h, h_{1}, \mathrm{~T}\right) * M_{r}\left(h, h_{1}, 2 \mathrm{~T}\right)}-1\right) \\
& +\eta_{3}\left(\frac{M_{r}(h, h, \mathrm{~T})}{M_{r}\left(h, h_{1}, 2 \mathrm{~T}\right)}-1+\frac{M_{r}\left(h_{1}, h_{1}, \mathrm{~T}\right)}{M_{r}\left(h, h_{1}, 2 \mathrm{~T}\right)}-1\right)+\eta_{4}\left(\frac{1}{M_{r}(h, h, \mathrm{~T})}-1+\frac{1}{M_{r}\left(h_{1}, h_{1}, \mathrm{~T}\right)}-1\right) \\
= & \eta_{1}\left(\frac{1}{M_{r}\left(h, h_{1}, \mathrm{~T}\right)}-1\right)+\eta_{2}\left(\frac{M_{r}\left(h, h_{1}, \mathrm{~T}\right)}{M_{r}\left(h, h_{1}, 2 \mathrm{~T}\right)}-1\right)+\eta_{3}\left(\frac{1}{M_{r}\left(h, h_{1}, 2 \mathrm{~T}\right)}-1+\frac{1}{M_{r}\left(h, h_{1}, 2 \mathrm{~T}\right)}-1\right) \\
\leq & \eta_{1}\left(\frac{1}{M_{r}\left(h, h_{1}, \mathrm{~T}\right)}-1\right)+\eta_{2}\left(\frac{M_{r}\left(h, h_{1}, \mathrm{~T}\right)}{M_{r}\left(h, h_{1}, \mathrm{~T}\right) * M_{r}\left(h_{1}, h_{1}, \mathrm{~T}\right)}-1\right) \\
& +\eta_{3}\left(\frac{1}{M_{r}\left(h, h_{1}, \mathrm{~T}\right) * M_{r}\left(h_{1}, h_{1}, \mathrm{~T}\right)}-1+\frac{1}{M_{r}\left(h, h_{1}, \mathrm{~T}\right) * M_{r}\left(h_{1}, h_{1}, \mathrm{~T}\right)}-1\right) \\
= & \left(\eta_{1}+2 \eta_{3}\right)\left(\frac{1}{M_{r}\left(h, h_{1}, \mathrm{~T}\right)}-1\right)=\left(\eta_{1}+2 \eta_{3}\right)\left(\frac{1}{M_{r}\left(\Gamma(h, g), \Gamma\left(h_{1}, g_{1}\right), \mathrm{T}\right)}-1\right) \\
\leq & \left(\eta_{1}+2 \eta_{3}\right)^{2}\left(\frac{1}{M_{r}\left(h, h_{1}, \mathrm{~T}\right)}-1\right) \leq \cdots \leq\left(\eta_{1}+2 \eta_{3}\right)^{J}\left(\frac{1}{M_{r}\left(h, h_{1}, \mathrm{~T}\right)}-1\right) \longrightarrow 0, \quad \text { as } J \longrightarrow \infty .
\end{aligned}
$$

Hence, $M_{r}\left(h, h_{1}, \mathrm{~T}\right)=1 \Rightarrow h=h_{1}$ for $\mathrm{T} \gg 0$.

Corollary 2. Let $\Gamma: G \times G \longrightarrow G$ be a mapping on a complete FCMS $\left(G, M_{r}, *\right)$ in which $M_{r}$ is triangular and $\Gamma$ satisfies the inequality

$$
\begin{aligned}
\frac{1}{M_{r}(\Gamma(g, h), \Gamma(\xi, \kappa), \mathrm{T})}-1 \leq & \eta_{1}\left(\frac{1}{M_{r}(g, \xi, \mathrm{T})}-1\right)+\eta_{2}\left(\frac{M_{r}(g, \xi, \mathrm{T}) * M_{r}(\xi, \Gamma(\xi, \kappa), \mathrm{T})}{M_{r}(g, \Gamma(g, h), \mathrm{T}) * M_{r}(g, \Gamma(\xi, \kappa), 2 \mathrm{~T})}-1\right) \\
& +\eta_{4}\left(\frac{1}{M_{r}(g, \Gamma(g, h), \mathrm{T})}-1+\frac{1}{M_{r}(\xi, \Gamma(\xi, \kappa), \mathrm{T})}-1\right),
\end{aligned}
$$

$\forall g, h, \xi, \kappa \in G, \quad \mathrm{~T} \gg 0, \quad \eta_{1} \in(0,1), \quad$ and $\eta_{2}, \eta_{4} \geq 0$ with $\left(\eta_{1}+\eta_{2}+2 \eta_{4}\right)<1$. Then, $\Gamma$ has a unique coupled FP in $G$.
Corollary 3. Let $\Gamma: G \times G \longrightarrow G$ be a mapping on a complete FCMS $\left(G, M_{r}, *\right)$ in which $M_{r}$ is triangular and $\Gamma$ satisfies the inequality

$$
\begin{aligned}
\frac{1}{M_{r}(\Gamma(g, h), \Gamma(\xi, \kappa), \mathrm{T})}-1 \leq & \eta_{1}\left(\frac{1}{M_{r}(g, \xi, \mathrm{T})}-1\right) \\
& +\eta_{3}\left(\frac{M_{r}(g, \Gamma(g, h), \mathrm{T})}{M_{r}(g, \Gamma(\xi, \kappa), 2 \mathrm{~T})}-1+\frac{M_{r}(\xi, \Gamma(\xi, \kappa), \mathrm{T})}{M_{r}(g, \Gamma(\xi, \kappa), 2 \mathrm{~T})}-1\right) \\
& +\eta_{4}\left(\frac{1}{M_{r}(g, \Gamma(g, h), \mathrm{T})}-1+\frac{1}{M_{r}(\xi, \Gamma(\xi, \kappa), \mathrm{T})}-1\right),
\end{aligned}
$$

$\forall g, h, \xi, \kappa \in G, \quad \mathrm{~T} \gg 0, \quad \eta_{1} \in(0,1), \quad$ and $\eta_{3}, \eta_{4} \geq 0$ with $\left(\eta_{1}+2 \eta_{3}+2 \eta_{4}\right)<1$. Then, $\Gamma$ has a unique coupled FP in $G$.
Corollary 4. Let $\Gamma: G \times G \longrightarrow G$ be a mapping on a complete FCMS $\left(G, M_{r}, *\right)$ in which $M_{r}$ is triangular and $\Gamma$ satisfies the inequality

$$
\frac{1}{M_{r}(\Gamma(g, h), \Gamma(\xi, \kappa), \mathrm{T})}-1 \leq \eta_{1}\left(\frac{1}{M_{r}(g, \xi, \mathrm{T})}-1\right)+\eta_{4}\left(\frac{1}{M_{r}(g, \Gamma(g, h), \mathrm{T})}-1+\frac{1}{M_{r}(\xi, \Gamma(\xi, \kappa), \mathrm{T})}-1\right)
$$


$\forall g, h, \xi, \kappa \in G, \quad \mathrm{~T} \gg 0, \quad \eta_{1} \in(0,1), \quad$ and $\quad \eta_{4} \geq 0 \quad$ with $\left(\eta_{1}+2 \eta_{4}\right)<1$. Then, $\Gamma$ has a unique coupled FP in $G$.

Example 2. From Example 1, we define a FM $M_{r}: G \times G \times$ $(0, \infty) \longrightarrow[0,1]$ by

$$
M_{r}(g, h, \mathrm{~T})=\frac{\mathrm{T}}{\mathrm{T}+|(g-h) / 3|}, \quad \forall g, h \in G \text { and } \mathrm{T}>0 .
$$

Then, one can easily prove the triangular property of FCM from the above example and $\left(G, M_{r}, *\right)$ is a complete FCMS. We define a mapping $\Gamma: G \times G \longrightarrow G$ by

$$
\Gamma(g, h)= \begin{cases}\frac{2 g}{5}, & g, h \in[0,1], \\ \frac{2 g+h}{h}+1, & g, h \in[1, \infty) .\end{cases}
$$

Then,

$$
\frac{1}{M_{r}(\Gamma(g, h), \Gamma(\xi, \kappa), \mathrm{T})}-1=\frac{2}{5}\left(\frac{1}{M_{r}(g, \xi, \mathrm{T})}-1\right),
$$

$\forall g, h, \xi, \kappa \in G$ and $\mathrm{T} \gg 0$. Hence, we proved that a mapping $\Gamma$ is a coupled $f c-$ contractive. Now, By using Definition 4 (4) to simplify the $\eta_{2}$ rational term of (28), for $\mathrm{T} \gg 0$,

$$
\begin{aligned}
& \frac{M_{r}(g, \xi, \mathrm{T}) * M_{r}(\xi, \Gamma(\xi, \kappa), \mathrm{T})}{M_{r}(g, \Gamma(g, h), \mathrm{T}) * M_{r}(g, \Gamma(\xi, \kappa), 2 \mathrm{~T})}-1 \\
& \quad \leq \frac{M_{r}(g, \xi, \mathrm{T}) * M_{r}(\xi, \Gamma(\xi, \kappa), \mathrm{T})}{M_{r}(g, \Gamma(g, h), \mathrm{T}) * M_{r}(g, \xi, \mathrm{T}) * M_{r}(\xi, \Gamma(\xi, \kappa), \mathrm{T})}-1=\frac{1}{M_{r}(g, \Gamma(g, h), \mathrm{T})}=\frac{g}{5 \mathrm{~T}},
\end{aligned}
$$

$\forall g, h, \xi, \kappa \in G$. Again, by using Definition 4 (4) and (61) to simplify the $\eta_{3}$ rational term of (28), for $\mathrm{T} \gg 0$, we have

$$
\begin{aligned}
& \frac{M_{r}(g, \Gamma(g, h), \mathrm{T})}{M_{r}(g, \Gamma(\xi, \kappa), 2 \mathrm{~T})}-1+\frac{M_{r}(\xi, \Gamma(\xi, \kappa), \mathrm{T})}{M_{r}(g, \Gamma(\xi, \kappa), 2 \mathrm{~T})}-1 \\
& \quad \leq \frac{M_{r}(g, \Gamma(g, h), \mathrm{T})}{M_{r}(g, \Gamma(g, h), \mathrm{T}) * M_{r}(\Gamma(g, h), \Gamma(\xi, \kappa), \mathrm{T})}-1+\frac{M_{r}(\xi, \Gamma(\xi, \kappa), \mathrm{T})}{M_{r}(g, \xi, \mathrm{T}) * M_{r}(\xi, \Gamma(\xi, \kappa), \mathrm{T})}-1 \\
& \quad=\frac{1}{M_{r}(\Gamma(g, h), \Gamma(\xi, \kappa), \mathrm{T})}-1+\frac{1}{M_{r}(g, \xi, \mathrm{T})}-1=\frac{7}{5}\left(\frac{1}{M_{r}(g, \xi, \mathrm{T})}-1\right)=\frac{7}{15 \mathrm{~T}}|g-\xi|,
\end{aligned}
$$

$\forall g, h, \xi, \kappa \in G$. After simple routine calculation, we can get the $\eta_{4}$ term result of (28) as follows:

$$
\begin{aligned}
& \frac{1}{M_{r}(g, \Gamma(g, h), \mathrm{T})}-1 \\
& \quad+\frac{1}{M_{r}(\xi, \Gamma(\xi, \kappa), \mathrm{T})}-1=\frac{1}{5 \mathrm{~T}}|g+\xi|, \quad \text { for } \mathrm{T} \gg 0 .
\end{aligned}
$$

Hence, from the above, we conclude that all the conditions of the Theorem 2 are satisfied with $\eta_{1}=2 / 5, \eta_{2}=1 / 6$, $\eta_{3}=\eta_{4}=1 / 15$, and $\Gamma(g, h)=\Gamma(4,4)=4 \in[0, \infty)$.

\section{Application}

In this section, we present an application on Lebesgue integral-type contraction mapping to support our main work.
In 2002, Branciari proved the following result on complete metric space for a unique FP (see [36]).

Theorem 3. Let $(G, d)$ be a complete metric space, $\eta_{1} \in(0,1)$, and $\Gamma: G \longrightarrow G$ be a mapping such that for each $g, h \in G$,

$$
\int_{0}^{\mathrm{d}(\Gamma g, \Gamma h)} \varphi(\tau) \mathrm{d} \tau \leq \eta_{1} \int_{0}^{\mathrm{d}(g, h)} \varphi(\tau) \mathrm{d} \tau,
$$

where $\varphi:[0, \infty) \longrightarrow[0, \infty)$ is a Lebesgue integrable mapping which is summable (i.e., with finite integral on each compact subset of $[0, \infty))$ and for each $\kappa>0$,

$$
\int_{0}^{\kappa} \varphi(s) \mathrm{d} s>0 .
$$


Then, $\Gamma$ has a unique FP $u \in G$ such that for any $g \in G$, $\lim _{J \longrightarrow \infty} \Gamma^{J} g=u$.”
Theorem 4. Let $\Gamma: G \times G \longrightarrow G$ be a mapping on a complete FCMS $\left(G, M_{r}, *\right)$ in which $M_{r}$ is triangular and satisfies

Now, we are in the position to use the above concept and to prove a unique coupled FP theorem in complete FCMSs.

$$
\int_{0}^{\left(\left(1 /\left(M_{r}(\Gamma(g, h), \Gamma(\xi, \kappa), \tau)\right)\right)-1\right)} \varphi(\tau) \mathrm{d} \tau \leq \eta_{1} \int_{0}^{\left(\left(1 / M_{r}(g, \xi, \tau)\right)-1\right)} \varphi(\tau) \mathrm{d} \tau+\eta_{2} \int_{0}^{\left(\left(M_{r}(g, \xi, \mathrm{T}) /\left(M_{r}(g, \Gamma(g, h), \mathrm{T}) * M_{r}(\xi, \Gamma(g, h), 2 \mathrm{~T})\right)\right)-1\right)} \varphi(\tau) \mathrm{d} \tau
$$

for all $g, h, \xi, \kappa \in G, \quad \mathrm{\top} \gg 0, \quad \eta_{1} \in(0,1)$, and $\eta_{2} \geq 0$, and $\varphi:[0, \infty) \longrightarrow[0, \infty)$ is a Lebesgue integrable mapping which is summable (i.e., with finite integral on each compact subset of $[0, \infty))$ and for each $\kappa>0$,

$$
\int_{0}^{\kappa} \varphi(\tau) \mathrm{d} \tau>0
$$

Proof. Let any $g_{o}, h_{o} \in G$; we define sequences $\left\{g_{J}\right\}$ and $\left\{h_{J}\right\}$ in $G$ such that

$$
\Gamma\left(g_{J}, h_{J}\right)=g_{J+1}, \quad \text { and } \Gamma\left(h_{J}, g_{J}\right)=h_{J+1}, \text { for } J \geq 0 \text {. }
$$
$\mathrm{T} \gg 0$,

Then, $\Gamma$ has a unique coupled FP in $G$.

$$
\int_{0}^{\left(\left(1 /\left(M_{r}\left(g_{J}, g_{J+1}, \tau\right)\right)\right)-1\right)} \varphi(\tau) \mathrm{d} \tau=\int_{0}^{\left(\left(1 / M_{r}\left(\Gamma\left(g_{J-1}, h_{J-1}\right), \Gamma\left(g_{J}, h_{J}\right), \tau\right)\right)-1\right)} \varphi(\tau) \mathrm{d} \tau \leq \eta_{1} \int_{0}^{\left(\left(1 / M_{r}\left(g_{J-1}, g_{J}, \mathrm{\tau}\right)\right)-1\right)} \varphi(\tau) \mathrm{d} \tau
$$

Similarly, again by using the arguments, we have

$$
\begin{aligned}
& \int_{0}^{\left(\left(1 /\left(M_{r}\left(g_{J-1}, g_{J}, \mathrm{~T}\right)\right)\right)-1\right)} \varphi(\tau) \mathrm{d} \tau \\
& \quad \leq \eta_{1} \int_{0}^{\left(\left(1 / M_{r}\left(g_{J-2}, g_{J-1}, \mathrm{~T}\right)\right)-1\right)} \varphi(\tau) \mathrm{d} \tau, \quad \text { for } \mathrm{T} \gg 0 .
\end{aligned}
$$

Now, from (70) and (71) and by induction, for $\mathrm{T} \gg 0$, we have

$$
\begin{aligned}
\int_{0}^{\left(\left(1 /\left(M_{r}\left(g_{J}, g_{J+1}, \mathrm{~T}\right)\right)\right)-1\right)} \varphi(\tau) \mathrm{d} \tau & \leq \eta_{1} \int_{0}^{\left(\left(1 / M_{r}\left(g_{J-1}, g_{J}, \mathrm{~T}\right)\right)-1\right)} \varphi(\tau) \mathrm{d} \tau \\
& \leq \eta_{1}^{2} \int_{0}^{\left(\left(1 / M_{r}\left(g_{J-2}, g_{J-1}, \mathrm{~T}\right)\right)-1\right)} \varphi(\tau) \mathrm{d} \tau \\
& \leq \cdots \leq \eta_{1}^{J} \int_{0}^{\left(\left(1 / M_{r}\left(g_{0}, g_{1}, \mathrm{~T}\right)\right)-1\right)} \varphi(\tau) \mathrm{d} \tau \longrightarrow 0, \text { as } J \longrightarrow \infty
\end{aligned}
$$

This shows that $\left\{g_{J}\right\}$ is a $f c-$ contractive sequence, and therefore,

$$
\lim _{J \longrightarrow \infty} \int_{0}^{\left(\left(1 /\left(M_{r}\left(g_{J}, g_{J+1}, \mathrm{~T}\right)\right)\right)-1\right)} \varphi(\tau) \mathrm{d} \tau=0 \Rightarrow \lim _{J \longrightarrow \infty}\left(\frac{1}{M_{r}\left(g_{J}, g_{J+1}, \mathrm{~T}\right)}-1\right)=0, \quad \text { for } \mathrm{T} \gg 0
$$

Hence, we get that

$$
\text { Now, for } \ell>J \text { and for } \mathrm{T} \gg 0 \text {, }
$$

$$
\lim _{J \longrightarrow \infty} M_{r}\left(g_{J}, g_{J+1}, \mathrm{~T}\right)=1, \quad \text { for } \mathrm{T} \gg 0 .
$$




$$
\begin{aligned}
\int_{0}^{\left(\left(1 / M_{r}\left(g_{,}, g_{\ell, \tau}\right)\right)-1\right)} \varphi(\tau) \mathrm{d} \tau & \leq \int_{0}^{\left(\left(1 / M_{r}\left(g_{J}, g_{J+1}, \tau\right)\right)-1\right)} \varphi(\tau) \mathrm{d} \tau+\int_{0}^{\left(\left(1 /\left(M_{r}\left(g_{J+1}, g_{J+2}, \tau\right)\right)\right)-1\right)} \varphi(\tau) \mathrm{d} \tau+\cdots+\int_{0}^{\left(1 /\left(M_{r}\left(g_{\ell-1}, g_{\ell, \tau}\right)\right)-1\right)} \varphi(\tau) \mathrm{d} \tau \\
& \leq \eta_{1}^{J} \int_{0}^{\left(\left(1 / M_{r}\left(g_{0}, g_{1}, \tau\right)\right)-1\right)} \varphi(\tau) \mathrm{d} \tau+\eta_{1}^{J+1} \int_{0}^{\left(\left(1 / M_{r}\left(g_{0}, g_{1}, \tau\right)\right)-1\right)} \varphi(\tau) \mathrm{d} \tau+\cdots+\eta_{1}^{\ell-1} \int_{0}^{\left(\left(1 / M_{r}\left(g_{0}, g_{1}, \tau\right)\right)-1\right)} \varphi(\tau) \mathrm{d} \tau \\
& =\left(\eta-1^{J}+\eta_{1}^{J+1}+\cdots+\eta_{1}^{\ell-1}\right) \int_{0}^{\left(\left(1 / M_{r}\left(g_{0}, g_{1}, \tau\right)\right)-1\right)} \varphi(\tau) \mathrm{d} \tau \\
& =\frac{\eta_{1}^{J}}{1-\eta_{1}} \int_{0}^{\left(\left(1 / M_{r}\left(g_{0}, g_{1}, \tau\right)\right)-1\right)} \varphi(\tau) \mathrm{d} \tau \longrightarrow 0, \quad \text { as } J \longrightarrow \infty
\end{aligned}
$$

We get that

$$
\begin{aligned}
& \lim _{J \longrightarrow \infty} \int_{0}^{\left(\left(1 / M_{r}\left(g_{J}, g_{\ell}, T\right)\right)-1\right)} \varphi(\tau) \mathrm{d} \tau=0 \\
& \Rightarrow \lim _{J \longrightarrow \infty}\left(\frac{1}{M_{r}\left(g_{J}, g_{\ell}, T\right)}-1\right)=0, \quad \text { for } \mathrm{T} \gg 0 .
\end{aligned}
$$

Hence, $\left\{g_{J}\right\}$ is a Cauchy sequence. Since, by the completeness of $\left(G, M_{r}, *\right), \exists g \in G$ so that

$$
\lim _{J \longrightarrow \infty} M_{r}\left(g_{J}, g, T\right)=1, \quad \text { for } \mathrm{T} \gg 0 .
$$

Now, for sequence $\left\{h_{J}\right\}$ from (67) and from the proof of Theorem 1, for $T \gg 0$, we have

$$
\begin{aligned}
\int_{0}^{\left(\left(1 /\left(M_{r}\left(h_{J}, h_{J+1}, \tau\right)\right)\right)-1\right)} \varphi(\tau) \mathrm{d} \tau & =\int_{0}^{\left(\left(1 / M_{r}\left(\Gamma\left(h_{J-1}, g_{J-1}\right), \Gamma\left(h_{J}, g_{J}\right), t\right)\right)-1\right)} \varphi(\tau) \mathrm{d} \tau \\
& \leq \eta_{1} \int_{0}^{\left(\left(1 / M_{r}\left(h_{J-1}, h_{J}, \tau\right)\right)-1\right)} \varphi(\tau) \mathrm{d} \tau .
\end{aligned}
$$

Similarly, again by using the same arguments, we have $\int_{0}^{\left(\left(1 / M_{r}\left(h_{J-1}, h_{j}, \tau\right)\right)-1\right)} \varphi(\tau) \mathrm{d} \tau \leq \eta_{1} \int_{0}^{\left(\left(1 /\left(M_{r}\left(h_{J-2}, g_{J-1}, \tau\right)\right)\right)-1\right)} \varphi(\tau) \mathrm{d} \tau, \quad$ for $\mathrm{T} \gg 0$.

$$
\begin{aligned}
\int_{0}^{\left(\left(1 / M_{r}\left(h_{J}, h_{J+1}, \tau\right)\right)-1\right)} \varphi(\tau) \mathrm{d} \tau & \leq \eta_{1} \int_{0}^{\left(\left(1 / M_{r}\left(h_{J-1}, h_{J}, \tau\right)\right)-1\right)} \varphi(\tau) \mathrm{d} \tau \\
& \leq \eta_{1}^{2} \int_{0}^{\left(\left(1 /\left(M_{r}\left(h_{J-2}, g_{J-1}, \tau\right)\right)\right)-1\right)} \varphi(\tau) \mathrm{d} \tau \\
& \leq \cdots \leq \eta_{1}^{J} \int_{0}^{\left(\left(1 / M_{r}\left(h_{0}, h_{1}, \tau\right)\right)-1\right)} \varphi(\tau) \mathrm{d} \tau \longrightarrow 0, \quad \text { as } J \longrightarrow \infty
\end{aligned}
$$

This shows that $\left\{h_{J}\right\}$ is a $f c$-contractive sequence, and therefore,

$$
\lim _{J \longrightarrow \infty} \int_{0}^{\left(\left(1 / M_{r}\left(h_{J}, h_{J+1}, \tau\right)\right)-1\right)} \varphi(\tau) \mathrm{d} \tau=0 \Rightarrow \lim _{J \longrightarrow \infty}\left(\frac{1}{M_{r}\left(h_{J}, h_{J+1}, \mathrm{~T}\right)}-1\right)=0, \quad \text { for } \mathrm{T} \gg 0
$$

Hence, we get that 


$$
\lim _{J \longrightarrow \infty} M_{r}\left(h_{J}, h_{J+1}, \mathrm{~T}\right)=1, \quad \text { for } \mathrm{T} \gg 0 .
$$

Now, for $\ell>J$ and for $\mathrm{T} \gg 0$, we have

$$
\begin{aligned}
\int_{0}^{\left(\left(1 / M_{r}\left(h_{J}, h_{\ell}, \mathrm{T}\right)\right)-1\right)} \varphi(\tau) \mathrm{d} \tau & \leq \int_{0}^{\left(\left(1 / M_{r}\left(h_{J}, h_{J+1}, \mathrm{~T}\right)\right)-1\right)} \varphi(\tau) \mathrm{d} \tau+\int_{0}^{\left(\left(1 / M_{r}\left(h_{J+1}, h_{J+2}, \mathrm{~T}\right)\right)-1\right)} \varphi(\tau) \mathrm{d} \tau+\cdots+\int_{0}^{\left(\left(1 / M_{r}\left(h_{\ell-1}, h_{\ell}, \mathrm{T}\right)\right)-1\right)} \varphi(\tau) \mathrm{d} \tau, \\
& \leq \eta_{2}^{J} \int_{0}^{\left(\left(1 / M_{r}\left(h_{o}, h_{1}, \mathrm{~T}\right)\right)-1\right)} \varphi(\tau) \mathrm{d} \tau+\eta_{1}^{J+1} \int_{0}^{\left(\left(1 / M_{r}\left(h_{o}, h_{1}, \mathrm{~T}\right)\right)-1\right)} \varphi(\tau) \mathrm{d} \tau+\cdots+\eta_{1}^{\ell-1} \int_{0}^{\left(\left(1 / M_{r}\left(h_{o}, h_{1}, \mathrm{~T}\right)\right)-1\right)} \varphi(\tau) \mathrm{d} \tau \\
& =\left(\eta_{1}^{J}+\eta_{1}^{J+1}+\cdots+\eta_{1}^{\ell-1}\right) \int_{0}^{\left(\left(1 / M_{r}\left(h_{o}, h_{1}, \mathrm{~T}\right)\right)-1\right)} \varphi(\tau) \mathrm{d} \tau \\
& =\frac{\eta_{1}^{J}}{1-\eta_{1}} \int_{0}^{\left(\left(1 / M_{r}\left(h_{o}, h_{1}, \mathrm{~T}\right)\right)-1\right)} \varphi(\tau) \mathrm{d} \tau \longrightarrow 0, \quad \text { as } J \longrightarrow \infty .
\end{aligned}
$$

We get that

$$
\lim _{J \longrightarrow \infty} \int_{0}^{\left(\left(1 / M_{r}\left(h_{J}, h_{\ell}, \mathrm{T}\right)\right)-1\right)} \varphi(\tau) \mathrm{d} \tau=0 \Rightarrow \lim _{J \longrightarrow \infty}\left(\frac{1}{M_{r}\left(h_{J}, h_{\ell}, \mathrm{T}\right)}-1\right)=0, \quad \text { for } \mathrm{T} \gg 0 .
$$

Hence, $\left\{h_{J}\right\}$ is a Cauchy sequence. Since, by the completeness of $\left(G, M_{r}, *\right), \exists h \in G$ so that

$$
\lim _{J \longrightarrow \infty} M_{r}\left(h_{J}, h, \mathrm{~T}\right)=1, \quad \text { for } \mathrm{T} \gg 0 .
$$

$$
\begin{aligned}
\int_{0}^{\left(1 / M_{r}(g, \Gamma(g, h), \tau)\right)-1} & \leq \int_{0}^{\left(\left(1 / M_{r}\left(g, g_{J+1}, \tau\right)\right)-1\right)}+\int_{0}^{\left(\left(1 / M_{r}\left(\Gamma\left(g_{J}, h_{J}\right), \Gamma(g, h), \tau\right)\right)-1\right)}, \\
& \leq \int_{0}^{\left(\left(1 / M_{r}\left(g, g_{J+1}, \tau\right)\right)-1\right)}+\eta_{1} \int_{0}^{\left(\left(1 / M_{r}\left(g_{J}, g, \tau\right)\right)-1\right)}+\eta_{2} \int_{0}^{\left(\left(M_{r}\left(g_{J}, g, \tau\right) /\left(M_{r}\left(g_{J}, \Gamma\left(g_{J}, h_{J}\right), \tau\right) * M_{r}\left(g, \Gamma\left(g_{J}, h_{J}\right), 2 \tau\right)\right)\right)-1\right)} \\
& =\int_{0}^{\left(\left(1 / M_{r}\left(g, g_{J+1}, \tau\right)\right)-1\right)}+\eta_{1} \int_{0}^{\left(\left(1 / M_{r}\left(g_{J}, g, \tau\right)\right)-1\right)}+\eta_{2} \int_{0}^{\left(M_{r}\left(g_{J}, g, \tau\right) / M_{r}\left(g_{J}, g_{J+1}, \tau\right) * M_{r}\left(g, g_{J+1}, 2 T\right)-1\right)} \longrightarrow 0, \text { as } \longrightarrow \infty
\end{aligned}
$$

Now, we prove $\Gamma(g, h)=g$. Then, from (67), (74), and (77), for $\mathrm{T} \gg 0$,

Hence, $M_{r}(g, \Gamma(g, h), \tau)=1 \Rightarrow \Gamma(g, h)=g$ for $\mathrm{T} \gg 0$. Next, we shall prove that $\Gamma(h, g)=h$; again from (67), (82), and (85), for $\mathrm{T} \gg 0$, 


$$
\begin{aligned}
\int_{0}^{\left(1 / M_{r}(h, \Gamma(h, g), \tau)\right)-1} & \leq \int_{0}^{\left(\left(1 / M_{r}\left(h, h_{J+1}, \mathrm{~T}\right)\right)-1\right)}+\int_{0}^{\left(\left(1 / M_{r}\left(\Gamma\left(h_{J}, g_{J}\right), \Gamma(h, g), \mathrm{T}\right)\right)-1\right)}, \\
& \leq \int_{0}^{\left(\left(1 / M_{r}\left(h, h_{J+1}, \mathrm{~T}\right)\right)-1\right)}+\eta_{1} \int_{0}^{\left(\left(1 / M_{r}\left(h_{J}, h, \mathrm{~T}\right)\right)-1\right)}+\eta_{2} \int_{0}^{\left(\left(M_{r}\left(h_{J}, h, \mathrm{~T}\right) /\left(M_{r}\left(h_{J}, \Gamma\left(h_{J}, g_{J}\right), \mathrm{T}\right) * M_{r}\left(h, \Gamma\left(h_{J}, g_{J}\right), 2 \mathrm{~T}\right)\right)\right)-1\right)} \\
& =\int_{0}^{\left(\left(1 / M_{r}\left(h, h_{J+1}, \mathrm{~T}\right)\right)-1\right)}+\eta_{1} \int_{0}^{\left(\left(1 / M_{r}\left(h_{J}, h_{,}\right)\right)-1\right)}+\eta_{2} \int_{0}^{\left(\left(M_{r}\left(h_{J}, h_{,}\right) /\left(M_{r}\left(h_{J}, h_{J+1}, \mathrm{~T}\right) * M_{r}\left(h, h_{J+1}, 2 \mathrm{~T}\right)\right)\right)-1\right)} \longrightarrow 0, \text { as } J \longrightarrow \infty
\end{aligned}
$$

Hence, $M_{r}(h, \Gamma(h, g), \mathrm{T})=1$ which implies $\Gamma(h, g)=h$ for $T \gg 0$.

Uniqueness: suppose $\left(g_{1}, h_{1}\right)$ and $\left(h_{1}, g_{1}\right)$ are other coupled fixed-point pairs in $G \times G$ such that $\Gamma\left(g_{1}, h_{1}\right)=g_{1}$ and $\Gamma\left(h_{1}, g_{1}\right)=h_{1}$. Now, from (67) and from the proof of Theorem 1, for $\mathrm{T} \gg 0$,

$$
\begin{aligned}
\int_{0}^{\left(\left(1 / M_{r}\left(g, g_{1}, \tau\right)\right)-1\right)} \varphi(\tau) \mathrm{d} \tau & =\int_{0}^{\left(\left(1 / M_{r}\left(\Gamma(g, h), \Gamma\left(g_{1}, h_{1}\right), \mathrm{\tau}\right)\right)-1\right)} \varphi(\tau) \mathrm{d} \tau \\
& \leq \eta_{1} \int_{0}^{\left(\left(1 / M_{r}\left(g, g_{1}, \mathrm{~T}\right)\right)-1\right)} \varphi(\tau) \mathrm{d} \tau \\
& =\eta_{1} \int_{0}^{\left(\left(1 / M_{r}\left(\Gamma(g, h), \Gamma\left(g_{1}, h_{1}\right), \mathrm{T}\right)\right)-1\right)} \varphi(\tau) \mathrm{d} \tau \\
& \leq \eta_{1}^{2} \int_{0}^{\left(\left(1 / M_{r}\left(g, g_{1}, \mathrm{~T}\right)\right)-1\right)} \varphi(\tau) \mathrm{d} \tau \\
& \leq \cdots \leq \eta_{1}^{J} \int_{0}^{\left(\left(1 / M_{r}\left(g, g_{1}, \mathrm{~T}\right)\right)-1\right)} \varphi(\tau) \mathrm{d} \tau \longrightarrow 0, \quad \text { as } J \longrightarrow \infty
\end{aligned}
$$

Hence, we get that $M_{r}\left(g, g_{1}, \mathrm{~T}\right)=1 \Rightarrow g=g_{1}$ for $\mathrm{T} \gg 0$.

Next, we have to prove $h=h_{1}$, and now, by using (67) and

from the proof of Theorem 1 , for $\mathrm{T} \gg 0$, we have that

$$
\begin{aligned}
\int_{0}^{\left(1 /\left(M_{r}\left(h, h_{1}, \mathrm{~T}\right)\right)-1\right)} \varphi(\tau) \mathrm{d} \tau & =\int_{0}^{\left(1 / M_{r}\left(\Gamma(h, g), \Gamma\left(h_{1}, g_{1}\right), \mathrm{T}\right)-1\right)} \varphi(\tau) \mathrm{d} \tau \\
& \leq \eta_{1} \int_{0}^{\left(1 /\left(M_{r}\left(h, h_{1}, \mathrm{~T}\right)\right)-1\right)} \varphi(\tau) \mathrm{d} \tau \\
& =\eta_{1} \int_{0}^{\left(1 / M_{r}\left(\Gamma(h, g), \Gamma\left(h_{1}, g_{1}\right), \mathrm{T}\right)-1\right)} \varphi(\tau) \mathrm{d} \tau \\
& \leq \eta_{1}^{2} \int_{0}^{\left(1 /\left(M_{r}\left(h, h_{1}, \mathrm{~T}\right)\right)-1\right)} \varphi(\tau) \mathrm{d} \tau \\
& \leq \cdots \leq \eta_{1}^{J} \int_{0}^{\left(1 /\left(M_{r}\left(h, h_{1}, \mathrm{~T}\right)\right)-1\right)} \varphi(\tau) \mathrm{d} \tau \longrightarrow 0, \quad \text { as } J \longrightarrow \infty
\end{aligned}
$$
$\mathrm{T} \gg 0$.

Hence, we get that $M_{r}\left(h, h_{1}, \mathrm{~T}\right)=1 \Rightarrow h=h_{1}$ for

\section{Conclusion}

We established the new concept of rational coupled fc-contraction mapping in FCMSs and proved some unique rational coupled FP theorems in FCMSs under the rational coupled fc-contraction conditions by using the "triangular property of fuzzy cone metric" with the help of some suitable examples to unify our work. In the last section, we presented an application of the Lebesgue integral-type coupled contraction theorem for unique rational coupled FP in complete FCMSs. By using this concept, one can prove more rational coupled-type fc-contraction results in complete FCMSs with different integral types of application to prove unique coupled FP results. 


\section{Data Availability}

Data sharing does not apply to this article as no data sets were generated or analyzed during the current study.

\section{Conflicts of Interest}

The authors declare that there are no conflicts of interest regarding the publication of this paper.

\section{Acknowledgments}

The authors are grateful to the Deanship of Scientific Research, King Saud University for funding through Vice Deanship of Scientific Research Chairs.

\section{References}

[1] L. A. Zadeh, "Fuzzy sets," Information and Control, vol. 8, no. 3, pp. 338-353, 1965.

[2] O. Kramosil and J. Michalek, "Fuzzy metric and statistical metric spaces," Kybernetika, vol. 11, pp. 336-344, 1975.

[3] M. Grabiec, "Fixed points in fuzzy metric spaces," Fuzzy Sets and Systems, vol. 27, no. 3, pp. 385-389, 1988.

[4] P. Balasubramaniam, S. Muralisankar, and R. P. Pant, "Common fixed points of four mappings in a fuzzy metric space," Journal of Fuzzy Mathematics, vol. 10, no. 1, pp. 379-384, 2002.

[5] Y. J. Cho, "Fixed points in fuzzy metric spaces," Journal of Fuzzy Mathematics, vol. 10, no. 4, pp. 949-962, 1997.

[6] J.-X. Fang, "On fixed point theorems in fuzzy metric spaces," Fuzzy Sets and Systems, vol. 46, no. 1, pp. 107-113, 1992.

[7] A. George and P. Veeramani, "On some results in fuzzy metric spaces," Fuzzy Sets and Systems, vol. 64, no. 3, pp. 395-399, 1994.

[8] V. Gregori and A. Sapena, "On fixed-point theorems in fuzzy metric spaces," Fuzzy Sets and Systems, vol. 125, no. 2, pp. 245-252, 2002.

[9] C. D. Bari and C. Vetro, "Fixed points, attractors and weak fuzzy contractive mappings in a fuzzy metric space," Journal of Fuzzy Mathematics, vol. 1, pp. 973-982, 2005.

[10] F. Kiany and A. Amini-Haradi, "Fixed point and endpoint theorems for set-valued fuzzy contraction mapps in fuzzy metric spaces," Fixed Point Theory and Applications, vol. 94, p. 9, 2011.

[11] M. Imdad and J. Ali, "Some common fixed point theorems in fuzzy metric spaces," Mathematical Communications, vol. 11, pp. 153-163, 2006.

[12] T. Som, "Some results on common fixed point in fuzzy metric spaces," Journal of the Mathematical Society of Japan, vol. 33, pp. 553-561, 2007.

[13] S. U. Rehman, R. Chinram, and C. Boonpok, "Rational type fuzzy-contraction results in fuzzy metric spaces with an application," Jurnal Matematika, vol. 2021, Article ID 6644491, 13 pages, 2021.

[14] D. S. Jaggi, "Some unique fixed point theorems," Indian Journal of Pure and Applied Mathematics, vol. 8, pp. 223-230, 1977.

[15] J. Harjani, B. Lopez, and K. Sadarangani, “A fixed point theorem for mappings satisfying a contractive condition of rational type on a partially ordered metric space," Abstract and Applied Analysis, vol. 2010, Article ID 190701, 8 pages, 2010.

[16] N. V. Luong and N. X. Thuan, "Fixed point theorem for generalized weak contractions satisfying rational expressions in ordered metric spaces," Fixed Point Theory and Applications, vol. 2011, no. 1, p. 10, 2011.

[17] D. Guo and V. Lakshmikantham, "Coupled fixed points of nonlinear operators with applications," Nonlinear Analysis: Theory, Methods \& Applications, vol. 11, no. 5, pp. 623-632, 1987.

[18] T. G. Bhaskar and V. Lakshmikantham, "Fixed point theorems in partially ordered metric spaces and applications," Nonlinear Analysis: Theory, Methods \& Applications, vol. 65, no. 7, pp. 1379-1393, 2006.

[19] V. Lakshmikantham and L. Ćirić, "Coupled fixed point theorems for nonlinear contractions in partially ordered metric spaces," Nonlinear Analysis: Theory, Methods \& Applications, vol. 70, no. 12, pp. 4341-4349, 2009.

[20] S. Sedghi, I. Altun, and N. Shobe, "Coupled fixed point theorems for contractions in fuzzy metric spaces," Nonlinear Analysis, Theory, Methods and Applications, vol. 72, no. 3-4, pp. 1298-1304, 2010.

[21] L.-G. Huang and X. Zhang, "Cone metric spaces and fixed point theorems of contractive mappings," Journal of Mathematical Analysis and Applications, vol. 332, no. 2, pp. 1468-1476, 2007.

[22] M. Abbas and G. Jungck, "Common fixed point results for noncommuting mappings without continuity in cone metric spaces," Journal of Mathematical Analysis and Applications, vol. 341, no. 1, pp. 416-420, 2008.

[23] D. Ilic and V. Rakocevic, "Common fixed points for maps on cone metric space," Journal of Mathematical Analysis and Applications, vol. 341, pp. 876-882, 2008.

[24] P. Raja and S. M. Vaezpour, "Some extensions of Banach contraction principle in complete cone metric spaces," Fixed Point Theory and Applications, vol. 2008, Article ID 768294, 11 pages, 2008.

[25] S. Rezapour and R. Hamlbarani, "Some notes on the paper "Cone metric spaces and fixed point theorems of contractive mappings"” Journal of Mathematical Analysis and Applications, vol. 345, no. 2, pp. 719-724, 2008.

[26] T. Öner, M. B. Kandemir, and B. Tanay, "Fuzzy cone metric spaces," The Journal of Nonlinear Science and Applications, vol. 8, no. 5, pp. 610-616, 2015.

[27] S. Ur Rehman and H.-X. Li, "Fixed point theorems in fuzzy cone metric spaces," The Journal of Nonlinear Science and Applications, vol. 10, no. 11, pp. 5763-5769, 2017.

[28] A. M. Ali and G. R. Kanna, "Intuitionistic fuzzy cone metric spaces and fixed point theorems," International Journal of Applied Mathematics, vol. 5, pp. 25-36, 2017.

[29] S. Jabeen, S. Ur Rehman, Z. Zheng, and W. Wei, "Weakly compatible and Quasi-contraction results in fuzzy cone metric spaces with application to the Urysohn type integral equations," Advances in Difference Equations, vol. 2020, no. 1, p. 16, 2020.

[30] T. Oner, "On some results in fuzzy cone metric spaces," International Journal of Computer Science and Network Security, vol. 4, pp. 37-39, 2016.

[31] T. Oner, "On the metrizability of fuzzy cone metric spaces," International Journal of Applied Management Science, vol. 2, pp. 133-135, 2016.

[32] G. X. Chen, S. Jabeen, S. U. Rehman et al., "Coupled fixed point analysis in fuzzy cone metric spaces with application to nonlinear integral equations," Advances in Difference Equations, vol. 202025 pages, 2020.

[33] S. U. Rehman and H. Aydi, "Rational fuzzy cone contractions on fuzzy cone metric spaces with an application to Fredholm integral equations," Journal of Function Spaces, vol. 2021, Article ID 5527864, 13 pages, 2021. 
[34] M. T. Waheed, S. U. Rehman, N. Jan, A. Gumaei, and M. AlRakhami, "Some new coupled fixed-point findings depending on another function in fuzzy cone metric spaces with application," Mathematical Problems in Engineering, vol. 2021, Article ID 4144966, 21 pages, 2021.

[35] B. Schweizer and A. Sklar, Probabilistic Metric Spaces, NorthHolland Series, New York, NY, USA, 1983.

[36] A. Branciari, "A fixed point theorem for mappings satisfying a general contractive condition of integral type," International Journal of Mathematics and Mathematical Sciences, vol. 29, no. 9, pp. 531-536, 2002. 